\title{
Single Key Recovery Attacks on 9-round Kalyna-128/256 and Kalyna-256/512
}

\author{
Akshima, Donghoon Chang, Mohona Ghosh, Aarushi Goel, and Somitra Kumar Sanadhya \\ Indraprastha Institute of Information Technology, Delhi (IIIT-D), India \\ \{akshima12014, donghoon, mohonag, aarushi12003, somitra\}@iiitd.ac.in
}

\begin{abstract}
The Kalyna block cipher has recently been established as the Ukranian encryption standard in June, 2015. It was selected in a Ukrainian National Public Cryptographic Competition running from 2007 to 2010. Kalyna supports block sizes and key lengths of 128, 256 and 512 bits. Denoting the variants of Kalyna as Kalyna- $b / k$, where $b$ denotes the block size and $k$ denotes the keylength, the design specifies $k \in\{b, 2 b\}$. In this work, we re-evaluate the security bound of some reduced round Kalyna variants, specifically Kalyna-128/256 and Kalyna-256/512 against key recovery attacks in the single key model. We first construct new 6-round distinguishers and then use these distinguishers to demonstrate 9-round attacks on these Kalyna variants. These attacks improve the previous best 7-round attacks on the same.

Our 9-round attack on Kalyna-128/256 has data, time and memory complexity of $2^{105}, 2^{245.83}$ and $2^{226.86}$ respectively. For our 9-round attack on Kalyna-256/512, the data/time/memory complexities are $2^{217}, 2^{477.83}$ and $2^{443.45}$ respectively. The time and data complexities for Kalyna-256/512 reported in this work improve upon the previous best 7-round attack complexities on the same. The attacks presented in this work are currently the best on Kalyna. We apply multiset attack - a variant of meet-in-the-middle attack to achieve these results.
\end{abstract}

Keywords. Block cipher, Kalyna, Key Recovery, Differential enumeration, Single key model

\section{Introduction}

The block cipher Kalyna proposed by Oliynykov et al. has been recently selected as Ukranian encryption standard in 2015. The official Kalyna specification [11] defines three block sizes, i.e., 128-bit, 256-bit and 512-bit and three key sizes - 128-bit, 256-bit and 512-bit where key size can be equal to or double the block length. Consequently, if we denote a variant of Kalyna as Kalyna - $b / k$, where, $b$ and $k$ denote block size and key size respectively, then the five variants of Kalyna are: Kalyna - 128/128, Kalyna - 128/256, Kalyna - 256/256, Kalyna - 256/512 and Kalyna - 512/512. The number of rounds of these variants are - 10,14,14, 18 and 18 respectively. Kalyna block cipher adopts an SPN (substitution-permutation network) structure, similar to AES [2] but with increased MDS matrix size, a new set of four different S-boxes, pre-and post-whitening modular $2^{64}$ key addition and a new key scheduling algorithm.

The official version of Kalyna specification (in English) available publicly does not include any security analysis of the design. A preliminary study in [10], before this cipher was standardized, reports attack complexities for Kalyna -128/128 against various attacks such as differential, linear, integral, impossible differential, boomerang etc. and shows that upto 5 rounds of this variant can be broken. Similar results are claimed for other Kalyna variants as well. The designers of Kalyna thus claim brute force security against Kalyna for rounds $\geq 6$. In [1], AlTawy et al. presented the first detailed key recovery attack against standardized Kalyna-128/256 and Kalyna-256/512. They applied meet-in-the-middle (MITM) attack [6|5] to break 7-rounds of both Kalyna variants and demonstrated the best attack on Kalyna so far.

In this work, we extend the number of rounds attacked and show the first 9-round key recovery attack against Kalyna-128/256 and Kalyna-256/512. Similar to [1, our attack is inspired from the multiset attack demonstrated by Dunkelman et al. on AES in [6]. Multiset attack is a variant of meet-in-the-middle attack presented by Demirci et al. on AES in 4. Demirci et al.'s attack involves constructing a set of functions which map one active byte in the first round to another active byte after 4-rounds of AES. This set of functions depend on ' $\mathrm{P}$ ' parameters and can be described using a table of $2^{P}$ ordered 256-byte sequence of entries. This table is precomputed and stored, thus allowing building a 4-round distinguisher and attacking upto 8 rounds of AES. However, Demirci's attacks suffered from a very high memory complexity. To reduce the memory complexity of Demirci's attacks on AES, Dunkelman et al. in [6], proposed multiset attack which replaces the idea of storing 256 ordered byte sequences with 256 unordered byte sequences (with multiplicity). This reduced both memory and time complexity of MITM attack on AES by reducing the parameters to ' $\mathrm{Q}$ ' (where, $\mathrm{Q}<\mathrm{P}$ ). They also introduced the novel idea of differential 
enumeration technique to significantly lower the number of parameters required to construct the multiset from ' $Q$ ' to ' $\mathrm{R}$ ' (where, $\mathrm{R}<\mathrm{Q}<\mathrm{P}$ ), thus further decreasing the attack complexities on AES. Derbez at al. in [5] improved Dunkelman et al.'s attack on AES-192/256 by refining the differential enumeration technique. By using reboundlike techniques [8, they showed that the number of reachable multisets are much lower than those counted in Dunkelman et al.'s attack. This improvement allowed mounting of comparatively efficient attacks on AES and also enabled extension of number of rounds attacked. Due to structural similarities between Kalyna and AES, a similar attack was applied to 7-rounds of Kalyna by AlTawy et al. in [1. The multiset attack on AES-192/256 was further improved by $\mathrm{Li}$ et al. in 9 . They extended the number of rounds attacked to 9 by introducing the concept of key sieving, where dependencies between the AES round keys were exploited to filter out the wrong states thus reducing the number of possible multisets. Recently, in [12], Li et al. demonstrated the most efficient multiset attack on AES-256. By exploiting some more key sieving properties and clever MixColumn properties, they extended the number of rounds attacked to 10. Since this line of work has produced the best results on AES and Kalyna has not yet received significant attention of the cryptanalysis community, we investigate the effectiveness of improved multiset attack on Kalyna in this work.

In our attacks, we examine Kalyna-128/256 and Kalyna-256/512. We construct new 6-round distinguishers for both the variants and use it to extend our attacks up to 9 rounds. For Kalyna-256/512, we significantly reduce the data and time complexities of the previous best 7-round attack on the same [1. The key schedule algorithm of Kalyna does not allow recovery of all subkeys or the master key from one subkey only unlike AES [2]. However, it allows recovery of odd-round keys from even-round keys and vice-versa. This property will be used by us in our attacks to reduce the attack complexities. To the best of our knowledge, our attacks are the first attacks on 9-round Kalyna-128/256 and Kalyna-256/512 respectively.

Our Contribution. The main contributions of this work are as follows:

- We present the first 9-round key recovery attack on Kalyna-128/256 and Kalyna-256/512.

- We apply multiset attack to construct new 6-round distinguishers on each of the above mentioned Kalyna variants.

- Our 9-round attack on Kalyna-128/256 has data/time/memory complexity of $2^{105}, 2^{245.83}$ and $2^{226.86}$ respectively.

- Our 9-round attack on Kalyna-256/512 has data/time/memory complexity of $2^{217}, 2^{477.83}$ and $2^{443.45}$ respectively. This improves upon the previous best attack [1] in terms of time and data complexity as well.

Our results are summarized in Table 1 .

Table 1. Comparison of cryptanalytic attacks on round reduced variants of Kalyna. The blank entries were not reported in [10. (The memory complexity header represents the number of 128-bit blocks for Kalyna-128 and 256-bit blocks for Kalyna-256 required to be stored in memory.)

\begin{tabular}{|c|c|c|c|c|c|c|}
\hline Algorithm & $\begin{array}{c}\text { Rounds } \\
\text { attacked }\end{array}$ & Attack & Time & Data & Memory & Reference \\
& 2 & Interpolation & - & - & - & {$[10]$} \\
\cline { 2 - 7 } & 3 & Linear Attack & $2^{52.8}$ & - & - & {$[10$} \\
\cline { 2 - 7 } Kalyna-128/128 & 4 & Differential & $2^{55}$ & - & - & {$[10]$} \\
\cline { 2 - 7 } & 4 & Boomerang & $2^{120}$ & - & - & {$[10]$} \\
\cline { 2 - 7 } & 5 & Impossible Differential & $2^{62}$ & - & $2^{66}$ & {$[10]$} \\
\cline { 2 - 7 } & 5 & Integral & $2^{97}$ & - & $2^{33+4}$ & {$[10]$} \\
\hline \multirow{3}{*}{ Kalyna-128/256 } & 7 & Meet-in-the-Middle & $2^{230.2}$ & $2^{89}$ & $2^{202.64}$ & {$[1]$} \\
\cline { 2 - 7 } & 9 & Meet-in-the-Middle & $2^{245.83}$ & $2^{105}$ & $2^{226.86}$ & This work, $\S[4]$ \\
\hline \multirow{2}{*}{ Kalyna-256/512 } & 7 & Meet-in-the-Middle & $2^{502.2}$ & $2^{233}$ & $2^{170}$ & {$[1]$} \\
\cline { 2 - 7 } & 9 & Meet-in-the-middle & $2^{477.83}$ & $2^{217}$ & $2^{443.45}$ & This work, $\S[5$ \\
\hline
\end{tabular}


Organization. In $\S 2$ we provide a brief description of Kalyna and the notations adopted throughout the work. In $\S 3$, we give details of our 6-round distinguisher for Kalyna 128/256 followed by $\S 4$ where we present our 9-round attack on the same. In $\S 5$, we describe our 6-round distinguisher for Kalyna-256/512 and in $\S 6$ we discuss our 9-round attack on the same.In $\S$ ??, we discuss some errors in the attack presented by Li et al. in [9. Finally in $\S 7$, we summarize and conclude our work.

\section{Preliminaries}

In this section, we first describe Kalyna and then mention the key notations and definitions used in our cryptanalysis technique to facilitate better understanding.

\subsection{Description of Kalyna}

As discussed in $\$ 1$, the block cipher Kalyna- $b / k$ has five variants namely - Kalyna-128/128, Kalyna-128/256, Kalyna - 256/256, Kalyna-256/512 and Kalyna-512/512 where, $b$ is the block size and $k$ is the key size. The 128-bit, 256-bit and 512-bit internal states are treated as a byte matrix of $8 \times 2$ size, $8 \times 4$ size and $8 \times 8$ size respectively where, the bytes are numbered column-wise (as shown in Fig. 1). The pre-whitening and post-whitening keys are added modulo $2^{64}$ to the plaintext and ciphertext respectively columnwise. ${ }^{1}$ Each internal round consists of 4 basic operations (as shown in Fig. 2):

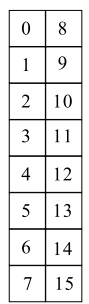

(a)

\begin{tabular}{|c|c|c|c|}
\hline 0 & 8 & 16 & 24 \\
\hline 1 & 9 & 17 & 25 \\
\hline 2 & 10 & 18 & 26 \\
\hline 3 & 11 & 19 & 27 \\
\hline 4 & 12 & 20 & 28 \\
\hline 5 & 13 & 21 & 29 \\
\hline 6 & 14 & 22 & 30 \\
\hline 7 & 15 & 23 & 31 \\
\hline
\end{tabular}

(b)

\begin{tabular}{|l|l|l|l|l|l|l|l|}
\hline 0 & 8 & 16 & 24 & 32 & 40 & 48 & 56 \\
\hline 1 & 9 & 17 & 25 & 33 & 41 & 49 & 57 \\
\hline 2 & 10 & 18 & 26 & 34 & 42 & 50 & 58 \\
\hline 3 & 11 & 19 & 27 & 35 & 43 & 51 & 59 \\
\hline 4 & 12 & 20 & 28 & 36 & 44 & 52 & 60 \\
\hline 5 & 13 & 21 & 29 & 37 & 45 & 53 & 61 \\
\hline 6 & 14 & 22 & 30 & 38 & 46 & 54 & 62 \\
\hline 7 & 15 & 23 & 31 & 39 & 47 & 55 & 63 \\
\hline
\end{tabular}

(c)

Fig. 1. (a)Byte numbering in a state of Kalyna128. (b) Byte numbering in a state of Kalyna-256. (c) Byte numbering in a state of Kalyna-512

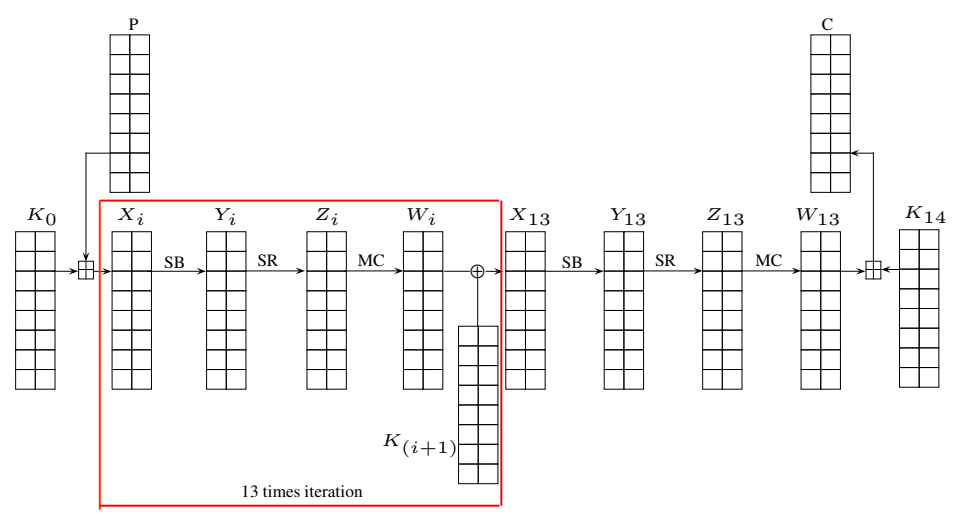

Fig. 2. One full encryption in Kalyna-128/256. (Refer to section 2.2 for notations)

1. SubBytes $(S B)$ - The nonlinear substitution layer uses four types of 8-bit S-boxes: $S_{0}, S_{1}, S_{2}$ and $S_{3}$. Each S-Box is defined to be an affine transformation of the inversion function over $\mathrm{GF}\left(2^{8}\right)$. Each byte $x$ of row $j$ (where $0 \leq j \leq 7$ ) in an intermediate state $s$ is substituted by $S_{j \text { mod } 4}(x)$.

2. Shift Rows (SR) - The linear shift rows operation performs circular right shift on each row of an internal state. The number of shifts $\left(\delta_{j}\right)$ in each row $j$ (where $0 \leq j \leq 7$ ) is calculated by $\delta_{j}=\left\lfloor\frac{j \cdot b}{512}\right\rfloor$, where $b$ denotes the block size. E.g., for row $j=6$ and block size $b=128$, the number of shifts $\left(\delta_{j}\right)=1$. The inverse shift rows operation circularly left shifts the elements by $\delta_{j}$ in each row.

3. MixColumn $(M C)$ - This linear transformation pre-multiplies each column of the state matrix by a $8 \times 8$ MDS matrix over $\mathrm{GF}\left(2^{8}\right)$. The vector $(0 \mathrm{x} 01,0 \mathrm{x} 01,0 \mathrm{x} 05,0 \mathrm{x} 01,0 \mathrm{x} 08,0 \mathrm{x} 06,0 \mathrm{x} 07,0 \mathrm{x} 04)$ forms the circulant MDS matrix for the MixColumn operation whereas the vector (0xAD, 0x95, 0x76, 0xA8, 0x2F, 0x49, 0xD7, 0xCA) forms the circulant MDS matrix for the inverse MixColumn operation. The branch factor of this MDS matrix is 9. The polynomial $x^{8}+x^{4}+x^{3}+x^{2}+1$ (represented as 0x11D in short) is used as the irreducible polynomial for Galois field multiplication. It is to be noted that unlike AES, in the last round of Kalyna, MixColumn operation is not omitted.

4. Add Round Key (ARK) - This step involves an exclusive-or operation with the round subkey. However, for the pre-whitening and post-whitening keys, key addition operation involves addition modulo $2^{64}$. The round subkeys are of the same size as the intermediate state size.

${ }^{1}$ For addition operation, little endian format is used, i.e., less significant bytes have smaller indices. E.g., if the intermediate state value is: $0 \times C D 0 \times 060 \times 050 \times 30 \times 170 \times 0 A \quad 0 \times 030 \times 02$ where, $0 \times C D$ is the most significant byte and $0 \times 02$ is the least significant byte. The representation in the little endian format would be $[0 \mathrm{x} 02,0 \mathrm{x} 03,0 \mathrm{x} 0 \mathrm{~A}, 0 \mathrm{x} 17,0 \mathrm{x} 30,0 \mathrm{x} 05,0 \mathrm{x} 06$, oxCD] corresponding to bytes $[0,1,2,3,4,5,6,7]$ of a column respectively. 
Key Scheduling Algorithm. The key scheduling algorithm of Kalyna first involves splitting of the master key $K$ into two parts - $K_{\alpha}$ and $K_{\omega}$. If the block size and key size are equal, i.e., $(k=b)$, then $K_{\alpha}=K_{\omega}=K$, otherwise if $(k=2 b)$, then $K_{\omega} \| K_{\alpha}=K$, i.e., $K_{\alpha}$ is set as $b / 2$ least significant bits of $K$ and $K_{\omega}$ is set as $b / 2$ most significant bits of $K$. Using these two parameters, an intermediate key $K_{\sigma}$ is generated which is then used to independently generate even indexed round keys. Complete details of the key schedule algorithm are not relevant to the attacks described in this work and hence are omitted here. One may refer to [11] for the same. Two properties which are important for us are as follows:

1. Recovery of a subkey does not allow recovery of master key better than brute force.

2. The keys for round $i$ where $i$ is an odd number can be linearly computed from the key used in round $(i-1)$ and vice- versa as follows:

$$
K_{i}=K_{i-1} \lll(b / 4+24)
$$

where, « denotes circular left shift operation.

\subsection{Notations and Definitions}

The following notations are followed throughout the rest of the paper.

\begin{tabular}{|c|c|}
\hline $\mathbf{P}$ & : Plaintext \\
\hline $\mathbf{C}$ & : Ciphertext \\
\hline$i$ & : Round number $i$, where, $0 \leq i \leq 8$ \\
\hline Kalyna- $b$ & : Kalyna with state size of $b$-bits \\
\hline Kalyna- $b / k$ & : Kalyna with state size of $b$-bits and key size of $k$-bits \\
\hline $\mathbf{K}_{\mathbf{i}}$ & : Subkey of round $i$ \\
\hline $\mathbf{U}_{\mathbf{i}}$ & $: M C^{-1}\left(\mathrm{~K}_{i}\right)$, where, $M C^{-1}$ is the inverse MixColumn operation \\
\hline $\mathbf{X}_{\mathbf{i}}$ & : State before SB in round $i$ \\
\hline $\mathbf{Y}_{\mathbf{i}}$ & : State before SR in round $i$ \\
\hline $\mathbf{Z}_{\mathbf{i}}$ & : State before MC in round $i$ \\
\hline $\mathbf{W}_{\mathbf{i}}$ & : State after MC in round $i$ \\
\hline$\Delta \mathrm{s}$ & : Difference in a state $\mathrm{s}$ \\
\hline $\mathbf{s}_{\mathbf{i}}[\mathbf{m}]$ & $\begin{array}{l}: m^{t h} \text { byte of state } \mathrm{s} \text { in round } i \text {, where, } 0 \leq m \leq l \text { and } l=15 \text { for } \\
\text { Kalyna- } 128 / 256 \text { and } l=31 \text { for Kalyna- } 256 / 512\end{array}$ \\
\hline $\mathbf{s}_{\mathbf{i}}[\mathbf{p}-\mathbf{r}]$ & $\begin{array}{l}: p^{\text {th }} \text { byte to } r^{\text {th }} \text { byte (both inclusive) of state } \mathrm{s} \text { in round } i \text {, where, } \\
0 \leq p<r \leq l \text { and } l=15 \text { for Kalyna- } 128 / 256 \text { and } l=31 \text { for } \\
\text { Kalyna-256/512 }\end{array}$ \\
\hline
\end{tabular}

In some cases we are interested in interchanging the order of the MixColumn and Add Round Key operations. As these operations are linear, they can be swapped, by first xoring the intermediate state with an equivalent key and then applying the MixColummn operation (as shown in Appendix A in Figs. 7. 8). This is exactly similar to what one can do in AES [5]. As mentioned above, we denote the equivalent round key by $U_{i}=M C^{-1}\left(K_{i}\right)$.

We utilize the following definitions for our attacks.

Definition 1 ( $\delta$-list). We define the $\delta$-list as an ordered list of 25616 -byte (or 32 -byte) distinct elements that are equal in 15 (or 31) bytes for Kalyna-128 (or Kalyna-256). Each of the equal bytes are called as passive bytes whereas the one byte that takes all possible 256 values is called the active byte [2]. We denote the $\delta$-list as $\left(x^{0}, x^{1}\right.$, $x^{2}, \ldots, x^{255}$ ) where $x^{j}$ indicates the $j^{\text {th }} 128$-bit (or 256-bit) member of the $\delta$-list for Kalyna-128 (or Kalyna-256). As mentioned in the notations, $x_{i}^{j}[\mathrm{~m}]$ represents the $m^{t h}$ byte of $x^{j}$ in round $i$.

Definition 2 (Multiset). A multiset is a set of elements in which multiple instances of the same element can appear. A multiset of 256 bytes, where each byte can take any one of the 256 possible values, can have $\left(\begin{array}{c}2^{8}+2^{8}-1 \\ 2^{8}\end{array}\right) \approx 2^{506.17}$ different values. 2

Definition 3 (Super S-Box). The Kalyna Super S-box (denoted as SSB) can be defined similar to AES Super S-box [3]. For each 8-byte key, it produces a mapping between an 8-byte input array to an 8-byte output array. Formally, a two round Kalyna can be written as:

$$
S B \rightarrow S R \rightarrow M C \rightarrow A R K \rightarrow S B \rightarrow S R \rightarrow M C \rightarrow A R K
$$

${ }^{2}$ Count of multisets of cardinality $r$ with elements from a set with cardinality $n=\left(\begin{array}{c}n+r-1 \\ r\end{array}\right)$ 
or,

$$
S R \rightarrow \underbrace{S B \rightarrow M C \rightarrow A R K \rightarrow S B} \rightarrow S R \rightarrow M C \rightarrow A R K{ }^{3}
$$

Since, MixColumns operation operates on a column of the state, the above map $(S B \rightarrow M C \rightarrow A K \rightarrow S B)$ in Eq. 2 can be described as $d$ parallel instances of SSB, where $d=2,4$ and 8 for Kalyna-128, Kalyna- 256 and Kalyna-512 respectively.

Two important properties that will be used in our attacks are as follows:

Property 1a. (Kalyna S-box) For any given Kalyna S-box, say $S_{i}$ (where, $i=0,1,2$ or 3 ) and any non-zero input - output difference pair, say $\left(\Delta_{i n}, \Delta_{\text {out }}\right)$ in $F_{256} \times F_{256}$, there exists one solution in average, say $y$, for which the equation, $S_{i}(y) \oplus S_{i}\left(y \oplus \Delta_{i n}\right)=\Delta_{\text {out }}$, holds true.

Proof. On analyzing the difference distribution table of the 4 S-boxes, it was observed that the number of solutions $\mathrm{N}\left(\Delta_{\text {in }}-\Delta_{\text {out }}\right)$ for any given $\Delta_{i n}-\Delta_{\text {out }}$ is either $0,2,4$ or rarely 6 or 8 . For a given $\Delta_{i n}$ and its 256 possible $\Delta_{\text {out }}$, the fequency of the solutions also vary depending on the choice of $\Delta_{\text {in }}$. For any random $\left(\Delta_{\text {in }}-\Delta_{\text {out }}\right)$, the probability that on average a solution exists can be calculated as: $\frac{256-a}{256}\left(\frac{b}{256-a} \times 2+\frac{c}{256-a} \times 4+\frac{d}{256-a} \times 6\right.$ $+\frac{e}{256-a} \times 8$ ) where $\mathrm{a}, \mathrm{b}, \mathrm{c}, \mathrm{d}$ and e is the frequency of zeroes, twos, fours, sixes and eights respectively. Since $2 \mathrm{~b}$ $+4 \mathrm{c}+6 \mathrm{~d}+8 \mathrm{e}=256$, on average one solutions always exists.

E.g, in $S_{2}$, for $\Delta_{\text {in }}=0 \mathrm{xAF}$ and all $256 \Delta_{\text {out }}$, there are 150 zeroes, 86 twos, 18 fours and 2 six. Hence, the average number of solutions can be calculated as: $\frac{256-150}{256}\left(\frac{86}{106} \times 2+\frac{18}{106} \times 4+\frac{2}{106} \times 6\right)=1$. Similar analysis can be done for other $\Delta_{i n}$ in $S_{1}$ and all other 3 S-boxes, i.e., $S_{0}, S_{1}$ and $S_{3}$.

Property 1b. (Kalyna Super S-box) For any given Kalyna Super S-box, say $S S B$ and any non-zero input - output difference pair, say $\left(\Delta_{\text {in }}, \Delta_{\text {out }}\right)$ in $F_{2^{64}} \times F_{2^{64}}$, the equation, $S S B(z) \oplus S S B\left(z \oplus \Delta_{\text {in }}\right)=\Delta_{\text {out }}$ has one solution in average.

Property 2. (Kalyna MixColumns) If the values (or the differences) in any eight out of its sixteen input/output bytes of the Kalyna MixColumn operation are known, then the values (or the differences) in the other eight bytes are uniquely determined and can be computed efficiently. This is similar to AES MixColumn property stated in [12].

Proof. The Kalyna Mixcolumn works on a column of 8 bytes. Thus, the inputs and outputs of Kalyna MixColumn operation can be related through 8 equations. Therefore, out of 16 variables ( 8 input and 8 output), if 8 variables are known then the other 8 variables can be uniquely determined through the 8 equations. This is because as mentioned in [7, any sub-matrix of a MDS matrix is invertible which guarantees existence of an unique solution.

The time complexity of the attack is measured in terms of 9-round Kalyna encryptions required. The memory complexity is measured in units of $b$-bit Kalyna (where, $b=128$ or 256) blocks required.

\section{Construction of distinguisher for 6-round Kalyna-128/256}

In this section, we construct a distinguisher on the 6 -inner rounds of Kalyna-128/256. Before, we proceed further, we first establish the following relation for Kalyna-128/256. According to Property 2, we can form an equation using any 11 out of 16 input-output bytes in the Kalyna MixColumn operation. For any round $j$, where, $0 \leq j \leq 8$ :

$$
\begin{aligned}
0 \mathrm{xCA} \cdot Z_{j}[12] \oplus 0 \mathrm{xAD} \cdot 0 x Z_{j}[13] \oplus 0 \mathrm{x} 49 \cdot Z_{j}[14] \oplus 0 \mathrm{xD} 7 \cdot Z_{j}[15]= & 0 \mathrm{x} 94 \cdot W_{j}[8] \oplus 0 \mathrm{xB} 4 \cdot W_{j}[9] \oplus 0 \mathrm{x} 4 \mathrm{E} \cdot W_{j}[10] \oplus \\
& 0 \mathrm{x} 7 \mathrm{E} \cdot W_{j}[11] \oplus 0 \mathrm{xC} 0 \cdot W_{j}[13] \oplus 0 \mathrm{xDA} \cdot W_{j}[14] \oplus \\
& 0 \mathrm{xC5} \cdot W_{j}[15] \\
& \text { or, }
\end{aligned}
$$

$0 \mathrm{xCA} \cdot Z_{j}[12] \oplus 0 \mathrm{xAD} \cdot Z_{j}[13] \oplus 0 \mathrm{x} 49 \cdot Z_{j}[14] \oplus 0 \mathrm{xD} 7 \cdot Z_{j}[15]=0 \mathrm{x} 94 \cdot\left(K_{j}[8] \oplus X_{j+1}[8]\right) \oplus 0 \mathrm{xB} 4 \cdot\left(K_{j}[9] \oplus X_{j+1}[9]\right)$

$$
\begin{aligned}
& \oplus 0 \mathrm{x} 4 \mathrm{E} \cdot\left(K_{j}[10] \oplus X_{j+1}[10]\right) \oplus 0 \mathrm{x} 7 \mathrm{E} \cdot\left(K_{j}[11] \oplus X_{j+1}[11]\right) \\
& \oplus 0 \mathrm{xC} 0 \cdot\left(K_{j}[13] \oplus X_{j+1}[13]\right) \oplus 0 \mathrm{xDA} \cdot\left(K_{j}[14] \oplus X_{j+1}[14]\right) \\
& \oplus 0 \mathrm{xC} 5 \cdot\left(K_{j}[15] \oplus X_{j+1}[15]\right)
\end{aligned}
$$

\footnotetext{
${ }^{3}$ Note that Sub Bytes and Shift Row operations in the first round have been interchanged as these functions commute with each other
} 
where, $W_{j}=K_{j} \oplus X_{j+1}$. Derivation of Eq. 3 is shown in Appendix B. Let,

$$
\begin{aligned}
P_{j}= & 0 \mathrm{xCA} \cdot Z_{j}[12] \oplus 0 \mathrm{xAD} \cdot Z_{j}[13] \oplus 0 \mathrm{x} 49 \cdot Z_{j}[14] \oplus 0 \mathrm{xD} 7 \cdot Z_{j}[15] \\
Q_{j}= & 0 \mathrm{x} 94 \cdot X_{j+1}[8] \oplus 0 \mathrm{xB} 4 \cdot X_{j+1}[9] \oplus 0 \mathrm{x} 4 \mathrm{E} \cdot X_{j+1}[10] \oplus 0 \mathrm{x} 7 \mathrm{E} \cdot X_{j+1}[11] \oplus 0 \mathrm{xC} 0 \cdot X_{j+1}[13] \oplus \\
& 0 \mathrm{xDA} \cdot X_{j+1}[14] \oplus 0 \mathrm{xC} 5 \cdot X_{j+1}[15] \\
\text { Const }= & 0 \mathrm{x} 94 \cdot K_{j}[8] \oplus 0 \mathrm{xB} 4 \cdot K_{j}[9] \oplus 0 \mathrm{x} 4 \mathrm{E} \cdot K_{j}[10] \oplus 0 \mathrm{x} 7 \mathrm{E} \cdot K_{j}[11] \oplus 0 \mathrm{xC} 0 \cdot K_{j}[13] \oplus 0 \mathrm{xDA} \cdot K_{j}[14] \\
& \oplus 0 \mathrm{xC} 5 \cdot K_{j}[15]
\end{aligned}
$$

then, Eq. 4 can be rewritten as,

$$
P_{j}=Q_{j} \oplus \text { Const }
$$

Eq. 8 will be used to establish the distinguishing property as shown next.

\subsection{Distinguishing Property for Kalyna-128/256}

Given, a list of 256 distinct bytes $\left(M^{0}, M^{1}, \ldots, M^{255}\right)$, a function $f:\{0,1\}^{128} \mapsto\{0,1\}^{128}$ and a 120 -bit constant $T$, we define a multiset $v$ as follows :

$$
\begin{aligned}
C^{i}= & f\left(T \| M^{i}\right), \text { where }(0 \leq i \leq 255) \\
u^{i}= & 0 \mathrm{x} 94 \cdot C^{i}[8] \oplus 0 \mathrm{xB} 4 \cdot C^{i}[9] \oplus 0 \mathrm{x} 4 \mathrm{E} \cdot C^{i}[10] \oplus 0 \mathrm{x} 7 \mathrm{E} \cdot C^{i}[11] \oplus 0 \mathrm{xC} 0 \cdot C^{i}[13] \oplus \\
& 0 \mathrm{xDA} \cdot C^{i}[14] \oplus 0 \mathrm{xC} 5 \cdot C^{i}[15] \\
v= & \left\{u^{0} \oplus u^{0}, u^{1} \oplus u^{0}, \ldots, u^{255} \oplus u^{0}\right\}
\end{aligned}
$$

Note that, $\left(T\left\|M^{0}, T\right\| M^{1}, \ldots, T \| M^{255}\right)$ forms a $\delta$-list and atleast one element of $v$ (i.e., $u^{0} \oplus u^{0}$ ) is always zero.

Distinguishing Property. Let us consider $\mathcal{F}$ to be a family of permutations on 128-bit. Then, given any list of 256 distinct bytes $\left(M^{0}, M^{1}, \ldots, M^{255}\right)$, the aim is to find how many multisets $v$ (as defined above) are possible when, $f \stackrel{\$}{\leftarrow} \mathcal{F}$ and $T \stackrel{\$}{\leftarrow}\{0,1\}^{120}$.

In case, when $\mathcal{F}=$ family of all permutations on 128-bit and $f \stackrel{\&}{\leftarrow} \mathcal{F}$. Under such setting, since in the multiset $v$, we have 255 values (one element is always 0 ) that are chosen uniformly and independently from the set $\{0,1, \ldots, 255\}$, the total number of possible multisets $v$ are atmost $\left(\begin{array}{c}2^{8}-1+2^{8}-1 \\ 2^{8}-1\end{array}\right) \approx 2^{505.17}$.

In case, when $\mathcal{F}=6$-full rounds of Kalyna-128/256 and $f \stackrel{\&}{\leftarrow}$. Here, $f \stackrel{\$}{\leftarrow} \mathcal{F} \Leftrightarrow K \stackrel{\$}{\leftarrow}\{0,1\}^{256}$ and $f=E_{K}$. Let us consider the 6 inner rounds of Kalyna-128/256 as shown in Fig. 3 . Here, $C$ in Eq. 9 is represented by $X_{6}$ and Eq. 10 is defined as :

$$
\begin{aligned}
u^{i}= & 0 \mathrm{x} 94 \cdot X_{6}^{i}[8] \oplus 0 \mathrm{xB} 4 \cdot X_{6}^{i}[9] \oplus 0 \mathrm{x} 4 \mathrm{E} \cdot X_{6}^{i}[10] \oplus 0 \mathrm{x} 7 \mathrm{E} \cdot X_{6}^{i}[11] \oplus 0 \mathrm{xC} 0 \cdot X_{6}^{i}[13] \oplus 0 \mathrm{xDA} \cdot X_{6}^{i}[14] \\
& \oplus 0 \mathrm{xC} 5 \cdot X_{6}^{i}[15]
\end{aligned}
$$

It is to be noted that under this setting, for each $i$ where, $(0 \leq i \leq 255)$, Eq. 12 is same as Eq. 6 computed at round 5, i.e., $u^{i}=Q_{5}^{i}$. Now, we state the following Observation 1.

Observation 1. The multiset $v$ is determined by the following 52 single byte parameters only :

- $X_{1}^{0}[0-7]$ (8-bytes)

- $X_{2}^{0}[0-15]$ (16-bytes)

- $X_{3}^{0}[0-15]$ (16-bytes)

- $X_{4}^{0}[0-3,12$ - 15] (8-bytes)

- $X_{5}^{0}[4-7]$ (4-bytes) 
Thus, the total number of possible multisets is $2^{52 \times 8}=2^{416}$ since, each 52 -byte value defines one sequence.

Proof. In round 0 (in Fig. 3), the set of differences $\left\{X_{0}^{0}[15] \oplus X_{0}^{0}[15], X_{0}^{1}[15] \oplus X_{0}^{0}[15], \ldots, X_{0}^{255}[15] \oplus X_{0}^{0}[15]\right\}$ (or, equivalently the set of differences at $X_{0}[15]$ ) is known to the attacker as there are exactly 256 differences possible. This is so, because in the plaintext we make the most significant byte as the active byte. Hence, when the pre-whitening key is added (columnwise), the carry-bit in the most significant bit is ignored limiting the possible values (and the differences) at $X_{0}[15]$ to 256 only. Since S-box is injective, exactly 256 values exist in the set $\left\{Y_{0}^{0}[15] \oplus Y_{0}^{0}[15], Y_{0}^{1}[15] \oplus Y_{0}^{0}[15], \ldots, Y_{0}^{255}[15] \oplus Y_{0}^{0}[15]\right\}$. As Shift Row (SR), MixColumn (MC) and Add Round Key (ARK) are linear operations, the set of differences at $X_{1}[0-7]$ will be known to the attacker.

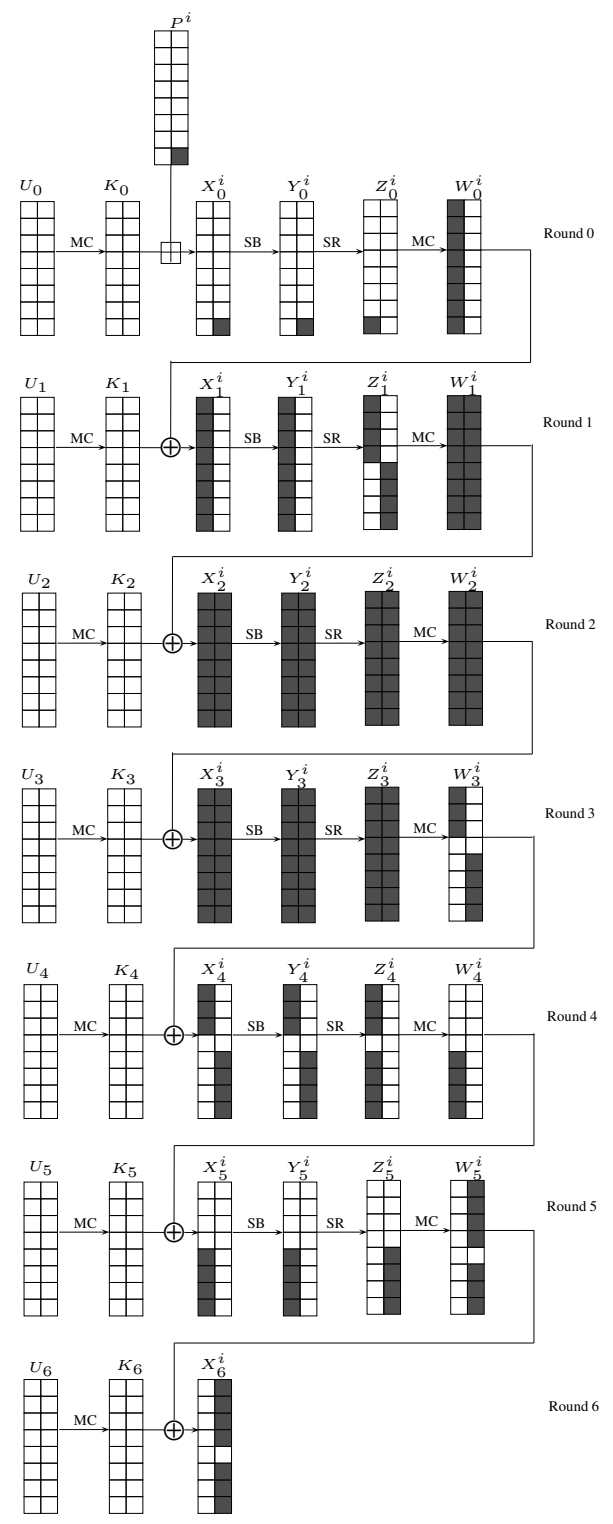

Fig. 3. 6-Round distinguisher in Kalyna-128/256 . Here, $P^{i}$ denotes $\left(T \| M^{i}\right)$ and $X_{j}^{i}, Y_{j}^{i}, Z_{j}^{i}, W_{j}^{i}$ denote intermediate states corresponding to $P^{i}$ in round j. The round subkeys $K_{j}$, where, $0 \leq j \leq 6$ are generated from the master key $K$.

Owing to the non-linearity of the S-box operation, the set of differences at $Y_{1}[0-7]$ cannot be computed to move forward. To allievate this problem, it is sufficient to guess $X_{1}^{0}[0-7]$, i.e., values of the active bytes of the first state (out of 256 states) at $X_{1}$ as it allows calculating the other $X_{1}^{i}[0-7]$ states (where, $1 \leq \mathrm{i} \leq 255$ ) and cross SB layer in round 1. Since, SR, MC and ARK operations are linear, the set of differences at $X_{2}[0-15]$ is known. Continuing 
in a similar manner as discussed above, if the attacker guesses full states $X_{2}^{0}[0-15]$ and $X_{3}^{0}$ [0-15], then the set of differences at $Z_{3}$, i.e., $\left\{Z_{3}^{0} \oplus Z_{3}^{0}, Z_{3}^{1} \oplus Z_{3}^{0}, \ldots, Z_{3}^{255} \oplus Z_{3}^{0}\right\}$ can be easily computed. Now at this stage, she can easily calculate the set of differences at $W_{3}[0,1,2,3,12,13,14,15]$ which is equal to the set of differences at $X_{4}[0,1,2,3,12,13,14,15] .{ }^{4}$. By guessing $X_{4}^{0}[0,1,2,3,12,13,14,15]$, the attacker can cross the SB layer in round 4 and calculate the set of differences at $W_{4}[4,5,6,7]$. By guessing $X_{5}^{0}[4,5,6,7]$, the attacker can obtain the set of values $\left\{Z_{5}^{0}[12-15], Z_{5}^{1}[12-15], \ldots, Z_{5}^{255}[12-15]\right\}$. Using these, she can compute $P_{5}^{i}$ at $Z_{5}^{i}$ as $P_{5}^{i}=$ $C A_{x} \cdot Z_{5}^{i}[12] \oplus A D_{x} \cdot Z_{5}^{i}[13] \oplus 49_{x} \cdot Z_{5}^{i}[14] \oplus D 7_{x} \cdot Z_{5}^{i}[15]$ (according to Eq. .5 ) and thus the set $\left\{P_{5}^{0} \oplus P_{5}^{0}, P_{5}^{0} \oplus P_{5}^{1}\right.$, $\left.\ldots, P_{5}^{255} \oplus P_{5}^{0}\right\}$. Since, according to Eq. $8 P_{j}^{i} \oplus P_{j}^{0}=\left(Q_{j}^{i} \oplus\right.$ Const $) \oplus\left(Q_{j}^{0} \oplus\right.$ Const $)=Q_{j}^{i} \oplus Q_{j}^{0}$ and $u^{i}=Q_{5}^{i}$ (mentioned above), the attacker can easily calculate the multiset $v=\left\{Q_{5}^{0} \oplus Q_{5}^{0}, Q_{5}^{1} \oplus Q_{5}^{0}, \ldots, Q_{5}^{255} \oplus Q_{5}^{0}\right\}$. This shows that the multiset $v$ depends on 52 parameters and can take $2^{416}$ possible values.

Since, there are $2^{416}$ possible multisets, if we precompute and store these values in a hash table, then the precomputation complexity goes higher than brute force for Kalyna-128/256. In order to reduce the number of multisets, we apply the Differential Enumeration technique suggested by Dunkelman et al. in [6] and improved by Derbez et al. in [5]. We call the improved version proposed in [5] as Refined Differential Enumeration.

Refined Differential Enumeration. The basic idea behind this technique is to choose a $\delta$-set such that several of the parameters mentioned in Observation 1 equal some pre-determined constants. To achieve so, we first construct a 6-round truncated differential trail in round 0 - round 5 (as shown in Fig. 3) where, the input difference is nonzero at one byte and output difference is non zero in 7 bytes. The probability of such a trail is $2^{-112}$ as follows: the one byte difference at $\Delta P[15]$ and correspondingly at $\Delta X_{0}[15]$ propagates to 8-byte difference in $\Delta X_{1}[0-7]$ and 16-byte difference in $\Delta X_{2}[0-15]$ and further till $\Delta Z_{3}[0-15]$ with probability 1 . Next, the probability that 16-byte difference in $\Delta Z_{3}[0-15]$ propagates to 7 -byte difference in $\Delta W_{3}[0-2,12-15]\left(=\Delta X_{4}[0-2,12-15]\right)$ is $2^{-72}$. This 7 -byte difference in $\Delta X_{4}$ propagates to 4 -byte difference in $\Delta W_{4}[4-7]$ followed by 7 -byte difference in $\Delta W_{5}[8-11,13-15]$ with a probability of $2^{-32}$ and $2^{-8}$ respectively. Thus, the overall probability of the differential from $\Delta P$ to $\Delta Z_{5}$ is $2^{-(72+32+8)}=2^{-112}$.

In other words, we require $2^{112}$ plaintext pairs to get a right pair. Once, we get a right pair, say $\left(P^{0}, P^{1}\right)$, we state the following Observation 2.

Observation 2. Given a right pair $\left(P^{0}, P^{1}\right)$ that follows the truncated differential trail shown in Fig. 3 the 52 parameters corresponding to $P^{0}$, mentioned in Observation 1 can take one of atmost $2^{224}$ fixed 52-byte values (out of the total $2^{416}$ possible values), where each of these $2^{224} 52$-byte values are defined by each of the $2^{224}$ values of the following 39 parameters:

- $\Delta Z_{0}[7](1$-byte)

- $X_{1}^{0}[0-7]$ (8-bytes)

- $Y_{3}^{0}[0-15](16$-bytes $)$

- $Y_{4}^{0}[0-3,12-15]$ (8-bytes)

- $Y_{5}^{0}[5-7]$ (3-bytes)

- $\Delta Z_{5}[12-14]$ (3-bytes)

Proof. Given a right pair $\left(P^{0}, P^{1}\right)$, the knowledge of these 39 new parameters allows us to compute all the differences shown in Fig. 3. This is so because the knowledge of $\Delta Z_{0}[7]$ allows us to compute $\Delta X_{1}[0-7]$. Then, if the values of $X_{1}^{0}[0-7]$ are known, one can compute the corresponding $X_{1}^{1}[0-7]$ and cross the S-box layer in round 1 to get $\Delta X_{2}$.

From the bottom side, it can be seen that $\Delta W_{5}[12]=\Delta Z_{5}[8]=\Delta Z_{5}[9]=\Delta Z_{5}[10]=\Delta Z_{5}[11]=0$. Thus, if $\Delta Z_{5}[12,13,14]$ are known, then using Property 2 (as 8 bytes are known), we can deduce $\Delta Z_{5}[15]$ (and $\Delta W_{5}$ [8-11, 13-15]). Knowledge of $\Delta Z_{5}[8-15]$ allows us to to compute $\Delta Y_{5}[4-7]$. Then, by guessing $Y_{5}^{0}[5-7]$, we can determine the corresponding $Y_{5}^{1}[5-7]$ and compute $\Delta X_{5}[5-7]$ (and $\Delta W_{4}[5-7]$ ). Now again, we know that $\Delta W_{4}[0]=\Delta W_{4}[1]=\Delta W_{4}[2]=\Delta W_{4}[3]=\Delta Z_{4}[3]=0$. Using Property 2 (as 8 bytes are known), we can deduce $\Delta W_{4}[4]$ (and $\Delta Z_{4}[0-2,4-7]$ ). This allows us to compute $\Delta X_{5}[4]$ as well. Since we already know $\Delta Y_{5}[4]$ (from $\Delta Z_{5}[12]$ guessed previously), using Property 1a., the possible values of $X_{5}[4]$ and $Y_{5}[4]$ can be computed.

Now, knowledge of $\Delta Z_{4}[0-7]$ allows us to compute $\Delta Y_{4}[0-3,12-15]$. By guessing $Y_{4}^{0}[0-3,12-15]$, we can obtain $\Delta Y_{3}[0-15]$. Using the value of $Y_{3}^{0}[0-15]$, we can compute $\Delta Y_{2}$. Then using Property 1a., the possible

\footnotetext{
${ }^{4}$ In Fig. 3 , byte 3 in states $W_{3}, X_{4}, Y_{4}$ and $Z_{4}$ have not been colored grey for a purpose which will be cleared when we reach Observation 2
} 
values of $X_{2}^{0}$ and $Y_{2}^{0}$ can be computed. At this stage, the total possible values of these 39 parameters are $2^{39 \times 8}=$ $2^{312}$.

However, for each value of this 39 -byte parameter, the following key bytes - $U_{2}[0-3,12-15], K_{3}, K_{4}[0-3,12-15]$ and $K_{5}[4-7]$ can be deduced as follows:

1. Knowledge of $X_{1}^{0}[0-7]$ allows us to compute the corresponding $Z_{1}^{0}[0-3,12-15]$. Xoring these values with $X_{2}^{0}[0-3,12-15]$ helps us in deducing $U_{2}[0-3,12-15]$.

2. Knowledge of $X_{2}^{0}$ allows us to compute the corresponding $W_{2}^{0}$. Xoring $W_{2}^{0}$ with $X_{3}^{0}$ helps us in deducing $K_{3}$.

3. Knowledge of $X_{3}^{0}$ and $X_{4}^{0}[0-3,12-15]$ (from $Y_{4}^{0}[0-3,12-15]$ ) can be used to deduce $K_{4}[0-3,12-15]$.

4. Knowledge of $X_{4}^{0}[0-3,12-15]$ and $X_{5}^{0}[4-7]$ (from $Y_{5}^{0}[4-7]$ ) helps us in deducing $K_{5}[4-7]$.

Now, according to the key schedule algorithm of Kalyna-128/256, from $K_{3}$, we can compute $K_{2}$ (according to Eq. 1) which allows us to compute the corresponding $U_{2}$. Thus, by comparing the computed $U_{2}[0-3,12-15]$ with the deduced $U_{2}[0-3,12-15]$, a sieve of 8-bytes (since matching probability is $2^{-64}$ ) can be applied to eliminate the wrong guesses. Similarly, again from Eq. 1 knowledge of $K_{5}[4-7]$ allows us to compute $K_{4}$ [12], $K_{4}[13]$ and $K_{4}[14]$ as $K_{4}[12]=K_{5}[5], K_{4}[13]=K_{5}[6]$ and $K_{4}[14]=K_{5}[7]$. This allows us a filtering of further 3 -bytes. Thus by key sieving, the total possible guesses of 39 -byte parameter reduces from $2^{39 \times 8}$ to $2^{(39-(8+3)) \times 8}=2^{28 \times 8}=2^{224}$.

Using Observation 1 and Observation 2, we state the following third Observation 3:

Observation 3. Given $\left(M^{0}, M^{1}, \ldots, M^{255}\right)$ and $f \stackrel{\$}{\leftarrow} \mathcal{F}$ and $T \stackrel{\$}{\leftarrow}\{0,1\}^{120}$, such that $T \| M^{0}$ and $T \| M^{j}$, (where, $j \in\{1, \ldots, 255\}$ ) is a right pair that follows the differential trail shown in Fig. 3, atmost $2^{224}$ multisets $v$ are possible.

Proof. From Observation 1, we know that each 52-byte parameter defines one multiset and Observation 2 restricts the possible values of these 52 -byte parameters to $2^{224}$. Thus, atmost $2^{224}$ multisets are only possible for Kalyna$128 / 256$.

As the number of multisets in case of 128 -bit random permutation $\left(=2^{505.17}\right)$ is much higher than 6-round Kalyna$128 / 256\left(=2^{224}\right)$, a valid distinguisher is constructed.

\section{Key Recovery Attack on 9-Round Kalyna-128/256}

In this section, we use our Observation 3 to launch meet-in-the-middle attack on 9-round Kalyna-128/256 to recover the key. The distinguisher is placed in round 0 to round 5 , i.e, the set of plaintexts is considered as the $\delta$-list with byte 15 being the active byte and the multiset sequence being checked at $X_{6}$ (as shown in Fig. 4 ). Three rounds are added at the bottom of the 6-round distinguisher. The attack consists of the following three phases:

\subsection{Precomputation Phase}

In this phase, we build a lookup table $T$ to store $2^{224}$ sequences to be used for comparison in the online phase. The construction of this table requires us to create two more hash tables $\left(T_{0}\right.$ and $\left.T_{1}\right)$ in the intermediate steps. The entire procedure is as follows:

1. For each $K_{3}$

- We guess $\Delta Z_{1}[0-3,12-15] \| \Delta X_{4}[0-2,12-15]$ to compute the difference $\Delta X_{2}$ and $\Delta Y_{3}$ respectively. We resolve $\left(\Delta X_{2}-\Delta Y_{3}\right)$ using Property $1 b$ to compute the corresponding $X_{2} \| X_{3}$. We then deduce $K_{2}$ from $K_{3}$ and compute the corresponding value of $Z_{1}[0-3,12-15]$. Using the guessed value of $\Delta Z_{1}[0-3,12-15]$ and the computed value of $Z_{1}[0-3,12-15]$, we compute $\Delta Z_{0}[0-7]$. If $\Delta Z_{0}[0-6]=0$ (which happens with a probability of $\left.2^{-56}\right)$, we store the corresponding $X_{1}[0-7]\left\|\Delta Z_{1}[0-3,12-15]\right\| X_{2}\left\|X_{3}\right\| W_{3}[12-$ $14] \| \Delta X_{4}[0-2,12-15]$ at index $K_{3}$ in table $T_{0}$. There are about $2^{64}$ entries for each index. 

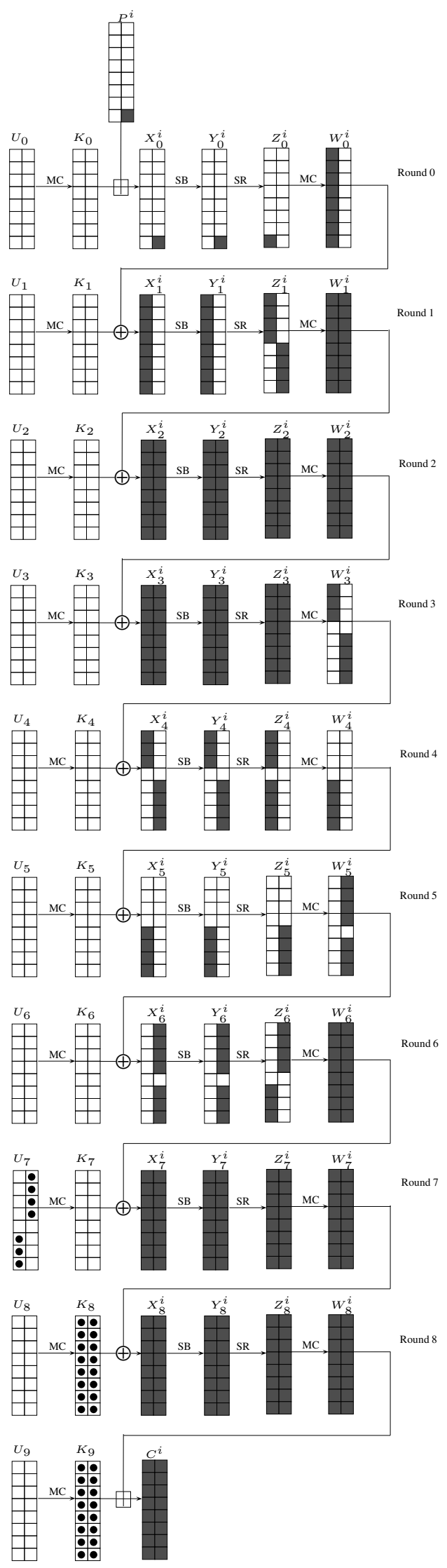

Fig. 4. 9-round attack on Kalyna-128/256. The subkey bytes guessed are shown dotted. 
2. For each guess of $\Delta Z_{5}[12-14]$

- We compute $\Delta Z_{5}[15]$ using Property 2.

- We guess $Y_{5}[5-7]$, compute $X_{5}[5-7]$ and $\Delta X_{5}[0-3,5-7]$ where, $\Delta X_{5}[0-3]=0$. Since, $\Delta X_{5}[0-3,5-7]=$ $\Delta W_{4}[0-3,5-7]$ and we know that $\Delta Z_{4}[3]=0$, thus we can compute $\Delta X_{5}[4]\left(=\Delta W_{4}[4]\right)$ and $\Delta Z_{4}[0-2,4-7]$ again using Property 2. Since $\Delta Y_{5}[4]$ is known from $\Delta Z_{5}[12]$, we can resolve $\left(\Delta X_{5}[4]-\Delta Y_{5}[4]\right)$ to get $X_{5}[4]$.

- We guess $Y_{4}[0-3,12-15]$ and compute corresponding $X_{4}[0-3,12-15]$ in the backward direction and $W_{4}[4-7]$ in the forward direction. This allows us to calculate $K_{5}[4-7]$ and deduce the corresponding $K_{4}[12-14]$. We use this to compute $W_{3}[12-14]$.

- We store $X_{4}[0-3,12-15]|| X_{5}[4-7]$ at index value $W_{3}[12-14] \| \Delta X_{4}[0-2,12-15]$ in table $T_{1}$. There are about $2^{32}$ entries for each index.

3. For each of the $2^{128}$ index of $K_{3}$ in table $T_{0}$, we have $2^{64}$ entries of $W_{3}[12-14] \| \Delta X_{4}[0-2,12-15]$ and corresponding to each of these we have $2^{32}$ entries of $X_{4}[0-3,12-15] \| X_{5}[4-7]$ in table $T_{1}$. So in all, after merging $T_{0}$ and $T_{1}$, we get $2^{128+64+32}=2^{224}$ unique set of 39 -byte parameters, that are required to construct the multiset $v$.

4. For each of these $2^{224} 39$-byte parameters, we calculate the corresponding 52-byte parameters for all the elements of the $\delta$-list and compute the multiset $v=\left\{u^{0} \oplus u^{0}, u^{1} \oplus u^{0}, \ldots, u^{255} \oplus u^{0}\right\}$. We store the multiset along with the 52 -byte parameters in the table $T$.

The time complexity to construct ${ }^{5} T_{0}=2^{(16+8+7) \times 8} \times 2^{-2.17}=2^{245.83}$. The time complexity to construct $T_{1}$ $=2^{(3+3+8) \times 8} \times 2^{-2.17}=2^{109.83}$. The time complexity to merge $T_{0}$ and $T_{1}=2^{128+64+32}=2^{224}$. Finally, the time complexity to construct $T=2^{224} \times 2^{8} \times 2^{-0.58}=2^{231.41}$.

\subsection{Online Phase}

In this phase we extend the differential trail described in Section 3, by adding 3 more rounds at the bottom (as shown in Fig. 4). The steps of the online phase are as follows:

1. We encrypt $2^{97}$ structures of $2^{8}$ plaintexts each where byte 15 takes all possible values and rest of the bytes are constants. We store the corresponding ciphertexts in the hash table.

2. For each of the $2^{112}\left(P_{0}, P_{0}^{\prime}\right)$ plaintext pairs, do the following:

- We guess $2^{128}$ values of $K_{9}$ and deduce the corresponding values of $K_{8}$ from $K_{9}$. We decrypt each of the ciphertext pairs through 2 rounds, to get $X_{7}$ and $\Delta X_{7}$. Then, we deduce the corresponding $\Delta W_{6}$ and $\Delta Z_{6}$.

- We filter out the keys, which do not give zero difference at $\Delta Z_{6}[0-4,12-15] .2^{56}$ key guesses are expected to remain.

- We pick one member of the pair, say $P_{0}$, create the $\delta$-list by constructing the rest of the 255 plaintexts as $P_{i}=P_{0} \oplus i$, where, $1 \leq i \leq 255$ and get their corresponding ciphertexts.

- For each remaining $2^{56}$ key guesses of $K_{8}$ and $K_{9}$, we guess $U_{7}[5-11]$, compute the corresponding $Z_{6}[5-11]$ and $Y_{6}[8-11,13-15]$ and then obtain the multiset $\left\{u^{0} \oplus u^{0}, u^{1} \oplus u^{0}, \ldots, u^{255} \oplus u^{0}\right\}$.

- We check whether this multiset exists in the precomputation table $T$ or not. If not, then we discard the corresponding guesses.

The probability for a wrong guess to pass the test is $2^{224} \times 2^{-467.6}=2^{-243.6}$. ${ }^{6}$ Since we try only $2^{112+56}=$ $2^{168}$ multisets, only the right subkey should verify the test.

\subsection{Recovering the remaining Subkey bytes}

The key schedule algorithm of Kalyna does not allow recovery of master key from any subkey better than bruteforce [11. However, knowledge of all round keys enables encryption/decryption. We follow a similar approach as described in [1] to recover all the round subkeys. When a match with a multiset is found using a given plaintextciphertext pair, we choose one of the ciphertexts and perform the following steps:

1. We already know the corresponding $K_{8}$ and $K_{9}$ and $U_{7}[5-11]$.

2. We guess the remaining 9 bytes of $U_{7}$, and deduce the corresponding $2^{72}$ values of $K_{7}$ and $K_{6}$.

${ }^{5}$ The normalization factor $2^{-2.17}$ is calculated by finding the ratio number of rounds encrypted/decrypted to 9 (i.e., the number of rounds of Kalyna considered in this paper). Similarly all other normalisation factors have been calculated.

${ }^{6}$ Note that the probability of randomly having a match is $2^{-467.6}$ and not $2^{-505.17}$ since the number of ordered sequences associated to a multiset is not constant [6]. 
3. For each $2^{72}$ guesses of $\left(K_{7}, K_{6}\right)$, from $X_{7}$ we compute $X_{5}$. We discard the key guesses for which $X_{5}[4-7]$ does not match with the values of $X_{5}[4-7]$ obtained from the corresponding matched multiset in the pre-computation table.

4. For the remaining $2^{72-32}=2^{40}$ guesses of $\left(K_{9}, K_{8}, K_{7}, K_{6}\right)$, we guess $2^{128}$ values of $K_{5}$. We deduce $X_{4}$ and discard the key guesses for which $X_{4}[0-2,12-15]$ does not match with the values obtained corresponding to the correct multiset sequence from the precomputation table. From a total of $2^{128+40}=2^{168}$ key guesses, $2^{112}$ key guesses are expected to remain.

5. We deduce $K_{4}$ from $K_{5}$ for the remaining key guesses and compute $X_{3}$. We compare this to the value obtained from the precomputation table corresponding to the correct multiset sequence and discard those that do not match. Only one value of $\left(K_{9}, K_{8}, K_{7}, K_{6}, K_{5}, K_{4}\right)$ is expected to remain.

6. One value of $K_{3}$ and $K_{2}$ corresponding to the matching sequence is already known from the pre-computation table. We deduce $X_{1}$ for the remaining one value of $\left(K_{9}, K_{8}, K_{7}, K_{6}, K_{5}, K_{4}, K_{3}, K_{2}\right)$.

7. We guess $2^{128}$ values of $K_{1}$, deduce $K_{0}$ and compute the plaintext. We compare this to the plaintext corresponding to ciphertext being decrypted. We are left with only one value of $\left(K_{9}, K_{8}, K_{7}, K_{6}, K_{5}, K_{4}, K_{3}, K_{2}\right.$, $\left.K_{1}, K_{0}\right)$.

Complexities. The time complexity of the precomputation phase is dominated by step 1 and is $2^{248} \times 2^{-2.17}=$ $2^{245.83}$ Kalyna-128/256 encryptions. The time complexity of the online phase is dominated by step 2 (part 1 ) and is $2^{112} \times 2^{128} \times 2^{-2.17}=2^{233.83}$. The time complexity of the Subkey recovery phase is dominated by step 4 which is $2^{168} \times 2^{-3.17}=2^{164.83}$. Clearly the time complexity of the whole attack is dominated by the time complexity of the precomputation phase, i.e., $2^{245.83}$. It was shown in [5] that each 256 -byte multiset requires 512-bits space. Hence, to store each entry in table $\mathrm{T}$, we require 512-bits to store the multiset and $52 \times 8=416$-bits to store the 52-byte parameters, i.e., a total of 928-bits $\left(=2^{9.86}\right)$. Therefore, the memory complexity of this attack is $2^{224} \times$ $2^{9.86-7}=2^{226.86}$ Kalyna 128 -bit blocks. The data complexity of this attack is $2^{105}$ plaintexts.

\section{Construction of distinguisher for 6-Round Kalyna-256/512}

In this section, we construct a distinguisher for the 6-inner rounds of Kalyna-256/512. The distinguisher construction details are similar to Kalyna-128/256 (discussed in Sec. 3) except the fact that here instead of counting multisets, we count 256-byte ordered sequences. The reason for opting ordered sequences would be discussed in Sec. 6 .

We first establish the following relation for Kalyna-256/512. According to Property 2, it is possible to construct an equation using any 12 out of 16 input-output bytes in the Kalyna MixColumn operation. For any round $j$, where, $0 \leq j \leq 8$ :

$$
\begin{aligned}
Z_{j}[8] \oplus Z_{j}[9] \oplus Z_{j}[12] \oplus Z_{j}[13]= & E A_{x} \cdot W_{j}[8] \oplus 54_{x} \cdot W_{j}[9] \oplus 7 D_{x} \cdot W_{j}[10] \oplus C 3_{x} \cdot W_{j}[11] \\
& \oplus E 0_{x} \cdot W_{j}[12] \oplus 5 E_{x} \cdot W_{j}[13] \oplus 7 D_{x} \cdot W_{j}[14] \\
& \oplus C 3_{x} \cdot W_{j}[15]
\end{aligned}
$$

Derivation of Eq. 13 is shown in Appendix C. Similar to as shown in Section 3, since, $W_{j}=K_{j} \oplus X_{j+1}$, if

$$
\begin{aligned}
P_{j}= & Z_{j}[8] \oplus Z_{j}[9] \oplus Z_{j}[12] \oplus Z_{j}[13] \\
Q_{j}= & E A_{x} \cdot X_{j+1}[8] \oplus 54_{x} \cdot X_{j+1}[9] \oplus 7 D_{x} \cdot X_{j+1}[10] \oplus C 3_{x} \cdot X_{j+1}[11] \oplus E 0_{x} \cdot X_{j+1}[12] \\
& \oplus 5 E_{x} \cdot X_{j+1}[13] \oplus 7 D_{x} \cdot X_{j+1}[14] \oplus C 3_{x} \cdot X_{j+1}[15] \\
\text { Const }= & E A_{x} \cdot K_{j}[8] \oplus 54_{x} \cdot K_{j}[9] \oplus 7 D_{x} \cdot K_{j}[10] \oplus C 3_{x} \cdot K_{j}[11] \oplus E 0_{x} \cdot K_{j}[12] \oplus 5 E_{x} \cdot K_{j}[13] \\
& \oplus 7 D_{x} \cdot K_{j}[14] \oplus C 3_{x} \cdot K_{j}[15]
\end{aligned}
$$

then, Eq. 13 can be rewritten as,

$$
P_{j}=Q_{j} \oplus \text { Const }
$$

\subsection{Construction of 6-round distinguisher for Kalyna-256/512}

Given a list of 256 distinct bytes $\left(M^{0}, M^{1}, \ldots, M^{255}\right)$, a function $f:\{0,1\}^{256} \mapsto\{0,1\}^{256}$ and a 248 -bit constant $\mathrm{T}$, we define an ordered sequence $o v$ as follows: 


$$
\begin{aligned}
C^{i}= & f\left(T \| M^{i}\right), \text { where }(0 \leq i \leq 255) \\
o u^{i}= & E A_{x} \cdot C^{i}[8] \oplus 54_{x} \cdot C^{i}[9] \oplus 7 D_{x} \cdot C^{i}[10] \oplus C 3_{x} \cdot C^{i}[11] \oplus E 0_{x} \cdot C^{i}[12] \oplus 5 E_{x} \cdot C^{i}[13] \\
& \oplus 7 D_{x} \cdot C^{i}[14] \oplus C 3_{x} \cdot C^{i}[15] \\
o v= & \left\{o u^{0} \oplus o u^{0}, o u^{1} \oplus o u^{0}, \ldots, o u^{255} \oplus o u^{0}\right\}
\end{aligned}
$$

Note that, $\left(T\left\|M^{0}, T\right\| M^{1}, \ldots, T \| M^{255}\right)$ forms a $\delta$-list and the first element of $o v$ (i.e., $o u^{0} \oplus o u^{0}$ ) is always zero.

Distinguishing Property. Let us consider $\mathcal{F}$ to be a family of permutations on 256-bit. Then, given any list of 256 distinct bytes $\left(M^{0}, M^{1}, \ldots, M^{255}\right)$, the aim is to find how many ordered sequences ov (as defined above) are possible when, $f \stackrel{\$}{\leftarrow} \mathcal{F}$ and $T \stackrel{\$}{\leftarrow}\{0,1\}^{248}$.

In case, when $\mathcal{F}=$ family of all permutations on 256-bit and $f \stackrel{\&}{\leftarrow} \mathcal{F}$. Under such setting, since, ov is a 256 -byte ordered sequence in which the first byte is always zero and the rest 255 bytes are chosen uniformly and independently from the set $\{0,1, \ldots, 255\}$, the total possible values of ov are $(256)^{255}=2^{2040}$.

In case, when $\mathcal{F}=6$-full rounds of Kalyna-256/512 and $f \stackrel{\&}{\leftarrow} \mathcal{F}$. Here, $f \stackrel{\$}{\leftarrow} \mathcal{F} \Leftrightarrow K \stackrel{\$}{\leftarrow}\{0,1\}^{512}$ and $f=E_{K}$. Let us consider the first 6 inner rounds of Kalyna-256/512 as shown in Fig. 5 . Here, $C$ in Eq. 18 is represented by $X_{6}$ and Eq. 19 is defined as :

$$
\begin{aligned}
o u^{i}= & E A_{x} \cdot X_{6}^{i}[8] \oplus 54_{x} \cdot X_{6}^{i}[9] \oplus 7 D_{x} \cdot X_{6}^{i}[10] \oplus C 3_{x} \cdot X_{6}^{i}[11] \oplus E 0_{x} \cdot X_{6}^{i}[12] \oplus 5 E_{x} \cdot X_{6}^{i}[13] \\
& \oplus 7 D_{x} \cdot X_{6}^{i}[14] \oplus C 3_{x} \cdot X_{6}^{i}[15]
\end{aligned}
$$

It is to be noted that here, for each $i$ where, $(0 \leq i \leq 255)$, Eq. 21 is same as Eq. 15 computed at round 5, i.e., $o u^{i}=Q_{5}^{i}$. Now, we state the following Observation 4 .

Observation 4. The ordered sequence ov is determined by the following 93 single byte parameters only :

- $X_{0}^{0}[31](1-$ byte $)$

- $X_{1}^{0}[16-23]$ (8-bytes)

- $X_{2}^{0}[0-31](32$-bytes $)$

- $X_{3}^{0}[0-31]$ (32-bytes)

- $X_{4}^{0}[2,3,6,7,8,9,12,13,18,19,22,23,24,25,28,29]$ (16-bytes)

- $X_{5}^{0}[8,9,28,29]$ (4-bytes)

Thus, the total number of ordered sequences is $2^{93 \times 8}=2^{744}$ since each 93 -byte value defines one sequence.

Proof. In round 0 (in Fig. 5), the ordered list of differences at $\left\{X_{0}^{0}[31] \oplus X_{0}^{0}[31], X_{0}^{1}[31] \oplus X_{0}^{0}[31], \ldots, X_{0}^{255}[31] \oplus\right.$ $\left.X_{0}^{0}[31]\right\}$ (or, equivalently the list of differences at $X_{0}[31]$ ) is known to the attacker as the list 7 of differences at $X_{0}[31]=$ list of differences at $P[31]$, i.e., $P^{i}[31] \oplus P^{0}[31]=X_{0}^{i}[31] \oplus X_{0}^{0}[31]$ for $(1 \leq i \leq 255)$. This is so, because in the plaintext, we make the most significant byte as the active byte. Hence, when the pre-whitening key is added (columnwise), the carry-bit in the most significant bit is ignored, thus converting the addition operation to xor operation. Since the value of $X_{0}^{0}[31]$ is known, the attacker can compute the other $X_{0}^{i}[31]$. This allows her to cross the $S B$ and $S R$ layer in round 0. Since, MixColumn (MC) and Add Round Key (ARK) are linear operations, the list of differences at $X_{1}[16-23]$ can be computed by the attacker.

Owing to the non-linearity of the S-box operation, the list of differences at $Y_{1}[16-23]$ cannot be computed to move forward. To allievate this problem, it is sufficient to guess $X_{1}^{0}[16-23]$ as it allows calculating other $X_{1}^{i}[16-23]$ states and cross SB layer in round 1. Since, SR, MC and ARK operations are linear, the list of differences at $X_{2}[0-31]$ is known. Continuing in a similar manner as discussed above, if the attacker guesses full states $X_{2}^{0}[0-31]$ and $X_{3}^{0}[0-31]$, then the list of differences at $Z_{3}$, i.e., $\left\{Z_{3}^{0} \oplus Z_{3}^{0}, Z_{3}^{1} \oplus Z_{3}^{0}, \ldots, Z_{3}^{255} \oplus Z_{3}^{0}\right\}$ can be easily computed.

\footnotetext{
${ }^{7}$ From now onwards, list denotes an ordered list
} 
This also allows her to calculate the list of differences at $X_{4}[2,3,6,7,8,9,12,13,18,19,22,23,24,25,28,29]$. By guessing $X_{4}^{0}[2,3,6,7,8,9,12,13,18,19,22,23,24,25,28,29]$, the attacker can cross the SB layer in round 4 and calculate the list of differences at $X_{5}[8,9,28,29]$. By guessing $X_{5}^{0}[8,9,28,29]$, the attacker can obtain the list of values $\left\{Z_{5}^{0}[8,9,12,13], Z_{5}^{1}[8,9,12,13], \ldots, Z_{5}^{255}[8,9,12,13]\right\}$. Using these, she can compute $P_{5}^{i}$ at $Z_{5}^{i}$ using Eq. 14 and thus the list $\left\{P_{5}^{0} \oplus P_{5}^{0}, P_{5}^{0} \oplus P_{5}^{1}, \ldots, P_{5}^{255} \oplus P_{5}^{0}\right\}$. Since, according to Eq. $17, P_{5}^{i} \oplus P_{5}^{0}=Q_{5}^{i} \oplus Q_{5}^{0}$ and $o u^{i}=Q_{5}^{i}$ (mentioned above), the attacker can easily calculate the ordered sequence $o v=\left\{Q_{5}^{0} \oplus Q_{5}^{0}, Q_{5}^{1} \oplus Q_{5}^{0}, \ldots, Q_{5}^{255} \oplus Q_{5}^{0}\right\}$. This shows that ov depends on 93 parameters and can take $2^{744}$ possible values. $\square$

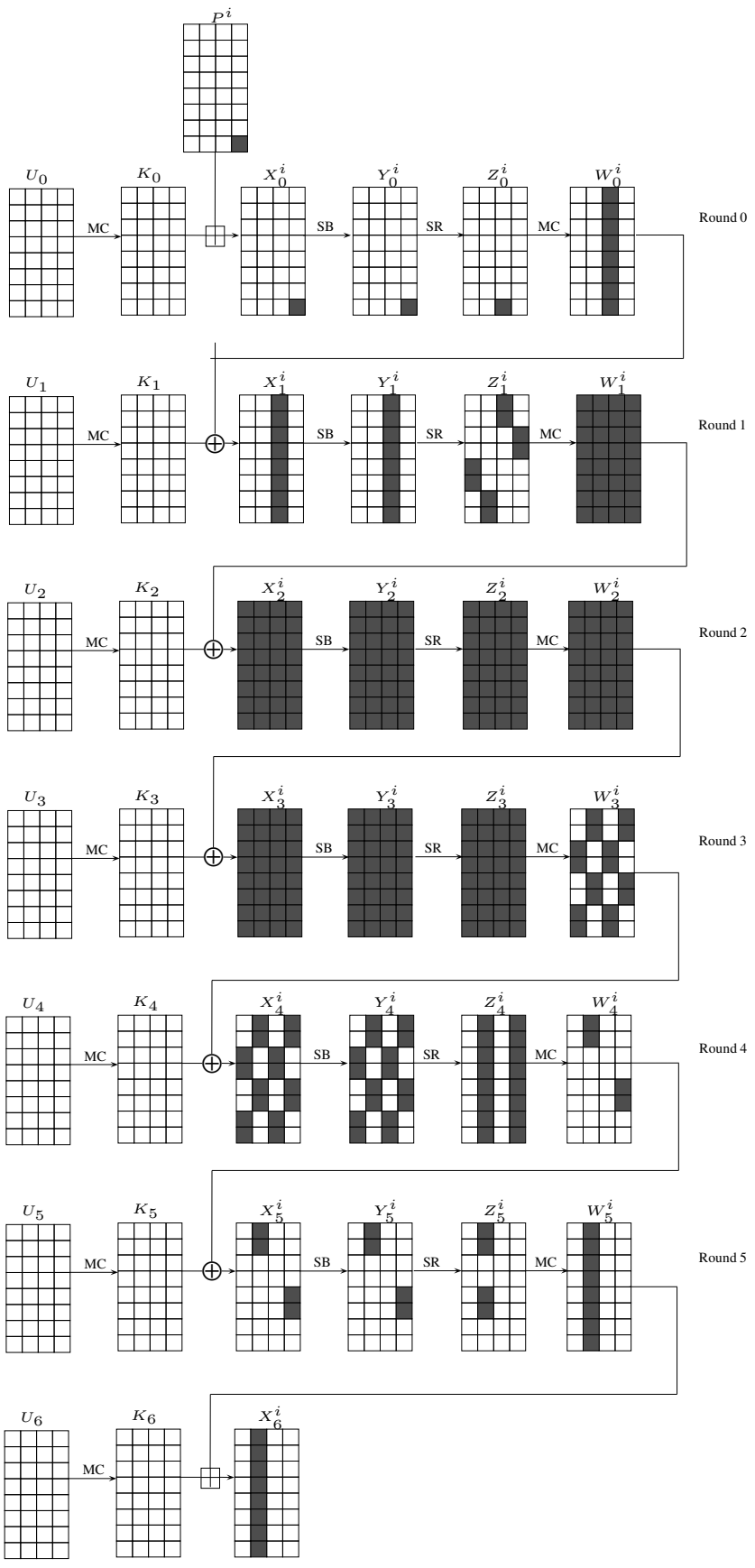

Fig. 5. 6-Round distinguisher in Kalyna-256/512. Here, $P^{i}$ denotes $\left(T \| M^{i}\right)$ and $X_{j}^{i}, Y_{j}^{i}, Z_{j}^{i}$, $W_{j}^{i}$ denote intermediate states corresponding to $P^{i}$ in round $\mathrm{j}$. The round subkeys $K_{j}$, where, $0 \leq j \leq 6$ are generated from the master key $K$.

Since, there are $2^{744}$ possible ordered sequences, if we precompute and store these values in a hash table, then the precomputation complexity goes higher than brute force for Kalyna-256/512. In order to reduce the number of ordered sequences, we apply the Refined Differential Enumeration technique as follows: 
Number of admissible ordered sequences. Consider the 6-round truncated differential trail in round 0 - round 5 (as shown in Fig. 5) where, the input difference is non-zero at one byte and output difference is non zero in 8 bytes. The probability of such a trail is $2^{-224}$ as follows: the one byte difference at $\Delta P[31]$ propagates to 32-byte difference in $\Delta Z_{3}[0-31]$ with probability 1 . Next, the probability that 32-byte difference in $\Delta Z_{3}[0-31]$ propagates to 16 -byte difference in $\Delta X_{4}[2,3,6,7,8,9,12,13,18,19,22,23,24,25,28,29]$ is $2^{-128}$. This 16-byte difference in $\Delta X_{4}$ propagates to 4 -byte difference in $\Delta W_{4}[8,9,28,29]$ followed by 8 -byte difference in $\Delta W_{5}[8-15]$ with a probability of $2^{-96}$. Thus, the overall probability of the differential trail from $\Delta P$ to $\Delta W_{5}$ is $2^{-(128+96)}=2^{-224}$.

In other words, we require $2^{224}$ plaintext pairs to get a right pair. Once, we get a right pair, say $\left(P^{0}, P^{1}\right)$, we state the following Observation 5.

Observation 5. Given a right pair $\left(P^{0}, P^{1}\right)$ that follows the truncated differential trail $\left(\Delta P \rightarrow \Delta W_{5}\right)$, the 93 parameters corresponding to $P^{0}$, mentioned in Observation 4 can take one of atmost $2^{440}$ fixed 93-byte values (out of the total $2^{744}$ possible values), where each of these $2^{440}$ 93-byte values are defined by each of the $2^{440}$ values of the following 66 parameters:

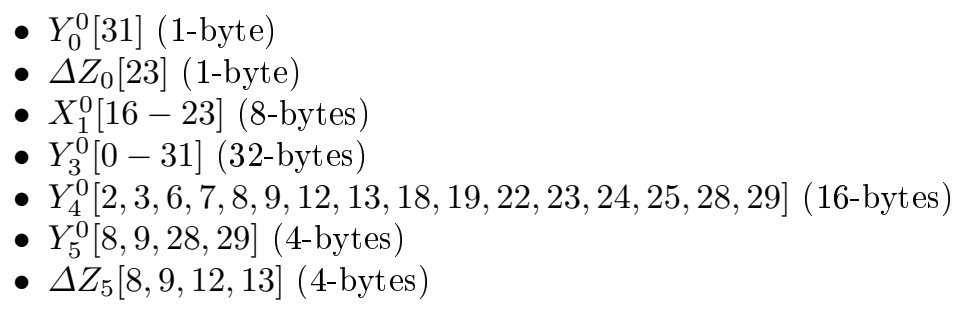

Proof. Given a right pair $\left(P^{0}, P^{1}\right)$, the knowledge of these 66 new parameters allows us to compute all the differences shown in Fig. 5as follows. Knowledge of $Y_{0}^{0}[31]$ allows us to compute $X_{0}^{0}[31]$. Knowing $\Delta Z_{0}[23]$ allows one to compute the difference $\Delta X_{1}[16-23]$. Then, if the values of $X_{1}^{0}[16-23]$ are known, one can compute the corresponding $X_{1}^{1}[16-23]$ and compute $\Delta X_{2}$.

From the bottom side, knowing $\Delta Z_{5}[8,9,12,13]$ allows one to compute $\Delta Y_{5}[8,9,28,29]$. Knowledge of $Y_{5}^{0}$ $[8,9,28,29]$ allows one to compute $Y_{5}^{1}[8,9,28,29]$, cross the SB layer in round 5 and obtain $\Delta Y_{4}^{0}[2,3,6,7,8,9,12$, $13,18,19,22,23,24,25,28,29]$. Proceeding in a similar manner, knowing $Y_{4}^{0}[2,3,6,7,8,9,12,13,18,19,22,23$, $24,25,28,29]$ and $Y_{3}^{0}[0-31]$ allows one to compute $\Delta Y_{2}^{0}[0-31]$. Then, using Property 1a., the possible values of $X_{2}^{0}$ and $Y_{2}^{0}$ can be computed. At this stage, the total possible values of these 65 parameters are $2^{66 \times 8}=2^{528}$.

However, for each value of this 66-byte parameter, the following key bytes - $U_{2}[4,5,14,15,16,17,26,27], K_{3}$, $K_{4}[2,3,6,7,8,9,12,13,18,19,22,23,24,25,28,29]$ and $K_{5}[8,9,28,29]$ can be deduced as follows:

1. Knowledge of $X_{1}^{0}[16-23]$ allows us to compute the corresponding $Z_{1}^{0}[4,5,14,15,16,17,26,27]$. Xoring these values with $X_{2}^{0}[4,5,14,15,16,17,26,27]$ helps us in deducing $U_{2}[4,5,14,15,16,17,26,27]$.

2. Knowledge of $X_{2}^{0}$ and $Y_{3}^{0}$ helps us in deducing $K_{3}$.

3. Knowledge of $Y_{3}^{0}$ and $Y_{4}^{0}[2,3,6,7,8,9,12,13,18,19,22,23,24,25,28,29]$ can be used to deduce $K_{4}[2,3,6,7,8$, $9,12,13,18,19,22,23,24,25,28,29]$.

4. Knowledge of $Y_{4}^{0}[2,3,6,7,8,9,12,13,18,19,22,23,24,25,28,29]$ and $Y_{5}^{0}[8,9,28,29]$ helps us in deducing $K_{5}$ $[8,9,28,29]$.

Now, according to the key schedule algorithm of Kalyna-128/256 from $K_{3}$, we can compute $K_{2}$ (according to Eq. 1) which allows us to compute the corresponding $U_{2}$. Thus, by comparing the computed $U_{2}[4,5,14,15,16,17$, $26,27]$ with the deduced $U_{2}[4,5,14,15,16,17,26,27]$, a sieve of 8 -bytes (since matching probability is $2^{-64}$ ) can be applied. Similarly, knowledge of $K_{4}[2,3,6,7,8,9,12,13,18,19,22,23,24,25,28,29]$ allows us to compute $K_{5}$ [8, 28, 29] which can then be matched with the deduced $K_{5}[8,28,29]$. This allows us a filtering of further 3 -bytes. Thus, by key sieving, a total of 11-byte filtering can be applied and the possible guesses of 66-byte parameter reduces from $2^{66 \times 8}$ to $2^{(66-11) \times 8}=2^{55 \times 8}=2^{440}$.

Using Observation 4 and Observation 5, we state the following third Observation 6:

Observation 6. Given $\left(M^{0}, M^{1}, \ldots, M^{255}\right)$ and $f \stackrel{\$}{\leftarrow} \mathcal{F}$ and $T \stackrel{\$}{\leftarrow}\{0,1\}^{248}$, such that $T \| M^{0}$ and $T \| M^{j}$, (where, $j \in\{1, \ldots, 255\}$ ) is a right pair that follows the differential trail shown in Fig. 5 , atmost $2^{440}$ multisets $v$ are possible. 
Proof. From Observation 4, we know that each 93-byte parameter defines one ordered sequence and Observation 5 restricts the possible values of these 93 -byte parameters to $2^{440}$. Thus, atmost $2^{440}$ ordered sequences are only possible for Kalyna-256/512.

As the number of ordered sequences in case of 256 -bit random permutation $\left(=2^{2040}\right)$ is much higher than 6-round Kalyna-256/512 $\left(=2^{440}\right)$, a valid distinguisher is therefore constructed.

\section{Key Recovery Attack on 9-Round Kalyna-256/512}

In this section, we use our Observation 6 to launch meet-in-the-middle attack on 9-round Kalyna-256/512 to recover the key. The distinguisher is placed in round 0 to round 5 (as shown in Fig. 6) and three rounds are added at the bottom of the 6 -round distinguisher. The attack consists of the following three phases:

\subsection{Precomputation Phase}

In this phase, we build a lookup table $T$ to store $2^{440}$ sequences to be used for comparison in the online phase. The construction of this table requires us to create two more hash tables $\left(T_{0}\right.$ and $\left.T_{1}\right)$ in the intermediate steps. The entire procedure is as follows:

1. For each $K_{3}$

- We guess $\Delta Z_{1}[4,5,14,15,16,17,26,27] \| \Delta X_{4}[2,3,6,7,8,9,12,13,18,19,22,23,24,25,28,29]$ to compute $\Delta X_{2}$ and $\Delta Y_{3}$ respectively. We resolve $\left(\Delta X_{2}-\Delta Y_{3}\right)$ using Property $1 b$ to compute the corresponding $X_{2} \| Y_{3}$. We then deduce $K_{2}$ from $K_{3}$ and compute the corresponding value of $Z_{1}[4,5,14,15,16,17,26,27]$. Using the guessed value of $\Delta Z_{1}[4,5,14,15,16,17,26,27]$ and the computed value of $Z_{1}[0-3,12-15]$, we compute $\Delta Z_{0}[16-23]$. If $\Delta Z_{0}[16-22]=0$ (which happens with a probability of $2^{-56}$ ), we store the corresponding $X_{1}[16-23]\left\|\Delta Z_{1}[4,5,14,15,16,17,26,27]\right\| X_{2}\left\|X_{3}\right\| W_{3}[7,8,19] \| \Delta X_{4}[2,3,6,7,8,9,12,13,18,19$, $22,23,24,25,28,29]$ at index $K_{3}$ in table $T_{0}$. There are about $2^{136}$ entries for each index.

2. For each guess of $\Delta Z_{5}[8,9,12,13] \| Y_{5}[8,9,28,29]$, compute $X_{5}[8,9,28,29], \Delta W_{4}[8,9,28,29]$ and $\Delta Y_{4}[2,3,6,7$, $8,9,12,13,18,19,22,23,24,25,28,29]$. Guess $Y_{4}[2,3,6,7,8,9,12,13,18,19,22,23,24,25,28,29]$ to compute $X_{4}[2,3,6,7,8,9,12,13,18,19,22,23,24,25,28,29]$ and $\Delta X_{4}[2,3,6,7,8,9,12,13,18,19,22,23,24,25,28,29]$ in the backward direction and $W_{4}[8,9,28,29]$ in the forward direction. From, $W_{4}[8,9,28,29]$ and $X_{5}[8,9,28,29]$ compute $K_{5}[8,9,28,29]$. Deduce $K_{4}[7,8,19]$ (where, $K_{5}[8]=K_{4}[19], K_{5}[28]=K_{4}[7]$ and $K_{5}[29]=K_{4}[8]$ ). Using, $X_{4}[7,8,19]$ and $K_{4}[7,8,19]$, compute $W_{3}[7,8,19]$.

3. For each entry of $W_{3}[7,8,19] \| \Delta X_{4}[2,3,6,7,8,9,12,13,18,19,22,23,24,25,28,29]$, we store $X_{4}[2,3,6,7,8,9$, $12,13,18,19,22,23,24,25,28,29] \| X_{5}[8,9,28,29]$ in a table $T_{1}$. There are $2^{40}$ entries per index.

4. For each of the $2^{256}$ index of $K_{3}$ in table $T_{0}$, we have $2^{136}$ entries of $W_{3}[7,8,19] \| \Delta X_{4}[2,3,6,7,8,9,12$, $13,18,19,22,23,24,25,28,29]$ and corresponding to each of these we have $2^{40}$ entries of $X_{4}[2,3,6,7,8,9$, $12,13,18,19,22,23,24,25,28,29] \| X_{5}[8,9,28,29]$ in table $T_{1}$. So in all, after merging $T_{0}$ and $T_{1}$, we get $2^{256+136+40}=2^{432}$ unique set of 65-byte parameters mentioned in Observation 5 .

5. For each guess of $X_{0}[31]$, combine the above merged entries with $X_{0}[31]$ to complete the set of 66-parameters mentioned in Observation 5. Now, there are a total of $2^{432+8}=2^{440}$ entries.

6. For each of these $2^{440} 66$-byte parameters, we calculate the corresponding 93 -byte parameters for all the elements of the $\delta$-list and compute the ordered sequence $o v=\left\{o u^{0} \oplus o u^{0}, o u^{1} \oplus o u^{0}, \ldots, o u^{255} \oplus o u^{0}\right\}$. We store the ordered sequence along with the 93 -byte parameters in the table $T$.

The time complexity to construct $T_{0}=2^{(32+8+16) \times 8} \times 2^{-2.17}=2^{445.83}$. The time complexity to construct $T_{1}$ $=2^{(4+4+16) \times 8} \times 2^{-2.17}=2^{189.83}$. The time complexity to merge $T_{0}$ and $T_{1}$ along with each guess of $X_{0}[31]=$ $2^{256+136+40+8}=2^{440}$. Finally, the time complexity to construct $T=2^{440} \times 2^{8} \times 2^{-0.58}=2^{447.42}$. Hence, overall time complexity is $2^{445.83}+2^{447.42} \approx 2^{447.83}$.

\subsection{Online Phase}

In this phase we extend the distinguisher in Section 5 , by adding 3 more rounds at the bottom (as shown in Fig. 6). The steps of the online phase are as follows:

1. We encrypt $2^{209}$ structures of $2^{8}$ plaintexts each where byte 31 takes all possible values and rest of the bytes are constants. We store the corresponding ciphertexts in the hash table. 

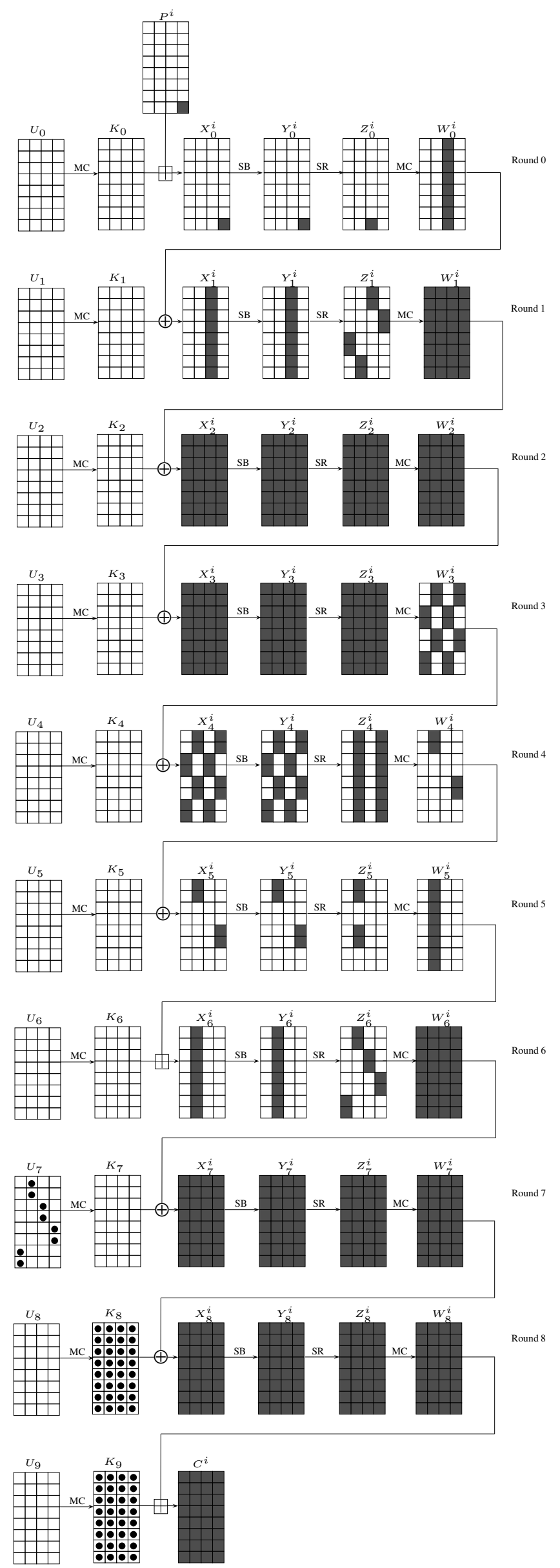

Fig. 6. 9-round attack on Kalyna-256/512. The subkey bytes guessed are shown dotted. 
2. For each of the $2^{224}\left(P_{0}, P_{0}^{\prime}\right)$ plaintext pairs, do the following:

- We guess $2^{256}$ values of $K_{9}$ and deduce the corresponding values of $K_{8}$ from $K_{9}$. We decrypt each of the ciphertext pairs through 2 rounds, to get $X_{7}$ and $\Delta X_{7}$. Then, we deduce the corresponding $\Delta W_{6}$ and $\Delta Z_{6}$.

- We filter out the keys, which do not give zero difference at $\Delta Z_{6}[0-5,10-17,20-27,30,31]$. This creates a filtering of $2^{-192}$ and hence only $2^{64}$ key guesses are expected to remain.

- We pick one member of the pair, say $P_{0}$, create the $\delta$-list by constructing the rest of the 255 plaintexts as $P_{i}=P_{0} \oplus i$, where, $1 \leq i \leq 255$ and get their corresponding ciphertexts.

- For each of the remaining $2^{64}$ key guesses of $K_{8}$ and $K_{9}$, we guess $U_{7}[6,7,8,9,18,19,28,29]$, compute the corresponding $Z_{6}[6,7,8,9,18,19,28,29]$ and $Y_{6}[8-15]$ and then obtain the ordered sequence $\left\{o u^{0} \oplus o u^{0}\right.$, $\left.o u^{1} \oplus o u^{0}, \ldots, o u^{255} \oplus o u^{0}\right\}$.

- We check whether this sequence exists in the precomputation table $T$ or not. If not, then we discard the corresponding guesses.

Reason for counting ordered sequences instead of multisets. The probability for a wrong guess to pass the test is $2^{440} \times 2^{-2040}=2^{-1600}$. Since we try only $2^{224+64}=2^{288}$ ordered sequences, only the right subkey should verify the test.

If we had opted for mutliset attack on Kalyna-256/512, the total possible admissible multisets would have been $2^{432}$ (as the parameter $X_{0}[31]$ would not have been required ). Therefore, the probability for a wrong guess to pass the test would have been $2^{432} \times 2^{-467.6}=2^{-35.6}$ (similar to that described in Section 4). As mentioned above, since we try $2^{288}$ multisets, we would have got mutliple candidates for the right subkey and unable to recover the secret key.

\subsection{Recovering the remaining Subkey bytes}

The remaining subkeys recovery process is similar to that discussed in Section 4.3 . When a match with an ordered sequence is found using a given plaintext-ciphertext pair, we choose one of the ciphertexts and perform the following steps:

1. We already know the corresponding $K_{8}$ and $K_{9}$ and $U_{7}[6,7,8,9,18,19,28,29]$.

2. We guess the remaining 24 bytes of $U_{7}$, and deduce the corresponding $2^{192}$ values of $K_{7}$ and $K_{6}$.

3 . For each $2^{192}$ guesses of $\left(K_{7}, K_{6}\right)$, from $X_{7}$ we compute $X_{5}$. We discard the key guesses for which $X_{5}[8,9,28,29]$ does not match with the values of $X_{5}[8,9,28,29]$ obtained from the corresponding matched ordered sequence in the pre-computation table.

4. For the remaining $2^{192-32}=2^{160}$ guesses of $\left(K_{9}, K_{8}, K_{7}, K_{6}\right)$, we guess $2^{256}$ values of $K_{5}$. We deduce $X_{4}$ and discard the key guesses for which $X_{4}[2,3,6,7,8,9,12,13,18,19,22,23,24,25,28,29]$ does not match with the values obtained corresponding to the correct ordered sequence from the precomputation table. From a total of $2^{160+256}=2^{416}$ key guesses, $2^{288}$ key guesses are expected to remain.

5. We deduce $K_{4}$ from $K_{5}$ for the remaining key guesses and compute $X_{3}$. We compare this to the value obtained from the precomputation table corresponding to the correct ordered sequence and discard those that do not match. $2^{32}$ values of $\left(K_{9}, K_{8}, K_{7}, K_{6}, K_{5}, K_{4}\right)$ are expected to remain.

6. One value of $K_{3}$ and $K_{2}$ corresponding to the matching sequence is already known from the pre-computation table. We deduce $X_{1}$ for the remaining $2^{32}$ values of $\left(K_{9}, K_{8}, K_{7}, K_{6}, K_{5}, K_{4}, K_{3}, K_{2}\right)$.

7. We guess $2^{256}$ values of $K_{1}$, deduce $K_{0}$ and compute $X_{0}$ [23] and plaintext. We compare this to $X_{0}[23]$ obtained from the precomputation table and to the plaintext corresponding to ciphertext being decrypted respectively. We are left with only $2^{24}$ values of $\left(K_{9}, K_{8}, K_{7}, K_{6}, K_{5}, K_{4}, K_{3}, K_{2}, K_{1}, K_{0}\right)$. We search these exhaustively to find the correct set of subkeys.

Complexities. The time complexity of the precomputation phase is $2^{447.83}$ Kalyna- $128 / 256$ encryptions. The time complexity of the online phase is dominated by step 2 (part 1) and is $2^{224} \times 2^{256} \times 2^{-2.17}=2^{477.83}$. The time complexity of the Subkey recovery phase is dominated by step 4 which is $2^{160} \times 2^{256} \times 2^{-3.17}=2^{412.83}$. Clearly the time complexity of the whole attack is dominated by the time complexity of the online phase, i.e., $2^{477.83}$. It was shown in [5] that each 256-byte multiset requires 512-bits space. Hence, to store each entry in table $\mathrm{T}$, we require 2048-bits to store the ordered sequence and $93 \times 8=744$-bits to store the 52 -byte parameters, i.e., a total of 928-bits $\left(=2^{11.45}\right)$. Therefore, the memory complexity of this attack is $2^{440} \times 2^{11.45-8}=2^{443.45}$ Kalyna 256-bit blocks. The data complexity of this attack is $2^{217}$ plaintexts. 


\section{Conclusions}

In this work, we utilize multiset attacks to launch key recovery attack on Kalyna-128/256 and Kalyna-256/512. We improve the previous 7-round attack on both the variants to demonstrate the first 9-round attacks on the same. Our attacks on Kalyna-256/512 even improve upon the previous 7-round attack on the same variant in terms of time and data complexities. We obtain these results by constructing new 6-round distinguishers on Kalyna and applying MITM attack on the rest of the rounds. Currently, this line of attack only works on Kalyna-b/2b variants and Kalyna variants in which block size and key size are equal appear to be safe. It would be an interesting problem to try applying multiset attacks on Kalyna-b/b. Presently, all five variants of Kalyna have been included in the Ukranian standard. However, our results as well as the previous 7-round attack show that compared to Kalyna-b/2b variants, Kalyna-b/b variants appear to be more robust.

\section{References}

1. Riham AlTawy, Ahmed Abdelkhalek, and Amr M. Youssef. A Meet-in-the-Middle Attack on Reduced-Round Kalynab/2b. IACR Cryptology ePrint Archive, 2015:762, 2015. http://eprint.iacr.org/2015/762

2. Joan Daemen and Vincent Rijmen. The Design of Rijndael: AES - The Advanced Encryption Standard. Information Security and Cryptography. Springer, 2002.

3. Joan Daemen and Vincent Rijmen. Understanding two-round differentials in AES. In Roberto De Prisco and Moti Yung, editors, Security and Cryptography for Networks, 5th International Conference, SCN 2006, Maiori, Italy, September 6-8, 2006, Proceedings, volume 4116 of Lecture Notes in Computer Science, pages 78-94. Springer, 2006.

4. Hüseyin Demirci and Ali Aydin Selçuk. A meet-in-the-middle attack on 8-round AES. In Kaisa Nyberg, editor, Fast Software Encryption, 15th International Workshop, FSE 2008, Lausanne, Switzerland, February 10-13, 2008, Revised Selected Papers, volume 5086 of Lecture Notes in Computer Science, pages 116-126. Springer, 2008.

5. Patrick Derbez, Pierre-Alain Fouque, and Jérémy Jean. Improved key recovery attacks on reduced-round AES in the single-key setting. In Thomas Johansson and Phong Q. Nguyen, editors, Advances in Cryptology - EUROCRYPT 2013, 32nd Annual International Conference on the Theory and Applications of Cryptographic Techniques, Athens, Greece, May 26-30, 2013. Proceedings, volume 7881 of Lecture Notes in Computer Science, pages 371-387. Springer, 2013.

6. Orr Dunkelman, Nathan Keller, and Adi Shamir. Improved single-key attacks on 8-round AES-192 and AES-256. J. Cryptology, 28(3):397-422, 2015.

7. Kishan Chand Gupta and Indranil Ghosh Ray. On constructions of MDS matrices from companion matrices for lightweight cryptography. In Alfredo Cuzzocrea, Christian Kittl, Dimitris E. Simos, Edgar R. Weippl, Lida Xu, Alfredo Cuzzocrea, Christian Kittl, Dimitris E. Simos, Edgar R. Weippl, and Lida Xu, editors, Security Engineering and Intelligence Informatics - CD-ARES 2013 Workshops: MoCrySEn and SeCIHD, Regensburg, Germany, September 2-6, 2013. Proceedings, volume 8128 of Lecture Notes in Computer Science, pages 29-43. Springer, 2013.

8. Mario Lamberger, Florian Mendel, Christian Rechberger, Vincent Rijmen, and Martin Schläffer. Rebound distinguishers: Results on the full whirlpool compression function. In Mitsuru Matsui, editor, Advances in Cryptology - ASIACRYPT 2009, 15th International Conference on the Theory and Application of Cryptology and Information Security, Tokyo, Japan, December 6-10, 2009. Proceedings, volume 5912 of Lecture Notes in Computer Science, pages 126-143. Springer, 2009.

9. Leibo Li, Keting Jia, and Xiaoyun Wang. Improved single-key attacks on 9-round AES-192/256. In Carlos Cid and Christian Rechberger, editors, Fast Software Encryption - 21st International Workshop, FSE 2014, London, UK, March 3-5, 2014. Revised Selected Papers, volume 8540 of Lecture Notes in Computer Science, pages 127-146. Springer, 2014.

10. Roman Oliynykov. Next Generation of Block Ciphers Providing High-Level Security, June 2015. http://http://www. slideshare.net/oliynykov/next-generation-ciphers/

11. Roman Oliynykov, Ivan Gorbenko, Oleksandr Kazymyrov, Victor Ruzhentsev, Oleksandr Kuznetsov, Yurii Gorbenko, Oleksandr Dyrda, Viktor Dolgov, Andrii Pushkaryov, Ruslan Mordvinov, and Dmytro Kaidalov. A new encryption standard of ukraine: The kalyna block cipher. IACR Cryptology ePrint Archive, 2015:650, 2015. http://eprint.iacr. org $/ 2015 / 650$

12. Li Rongjia and Jin Chenhui. Meet-in-the-middle attacks on 10-round AES-256. Designs, Codes and Cryptography, pages $1-13,2015$.

\section{A One round of Kalyna when MixColumn and Key Xoring operations are interchnaged}

Here, we show how one internal round of Kalyna works when MixColumn and AddRoundKey operations are interchanged. 


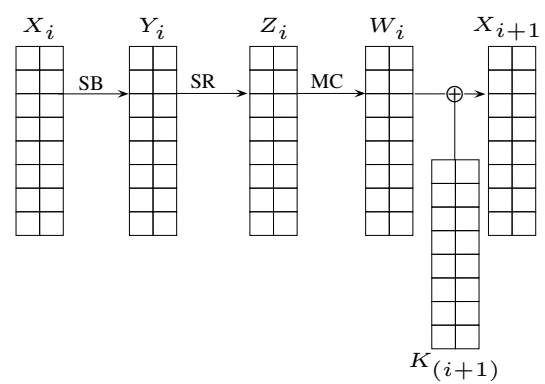

Fig. 7. Normal one round of Kalyna-128/128.

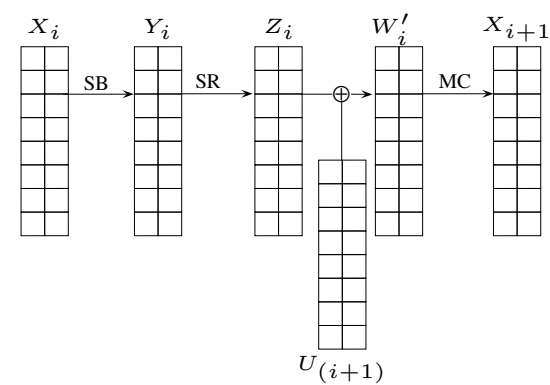

Fig. 8. One round of Kalyna-128/128 with swapped MC and ARK operation. Here, $W_{i}^{\prime}=Z_{i} \oplus U_{i+1}$.

\section{B Derivation of Eq. 3 defined in Section 3}

$$
\left(\begin{array}{cccccccc}
A D_{x} & 95_{x} & 76_{x} & A 8_{x} & 2 F_{x} & 49_{x} & D 7_{x} & C A_{x} \\
C A_{x} & A D_{x} & 95_{x} & 76_{x} & A 8_{x} & 2 F_{x} & 49_{x} & D 7_{x} \\
D 7_{x} & C A_{x} & A D_{x} & 95_{X} & 76_{x} & A 8_{x} & 2 F_{x} & 49_{x} \\
49_{x} & D 7_{x} & C A_{x} & A D_{x} & 95_{x} & 76_{x} & A 8_{x} & 2 F_{x} \\
2 F_{x} & 49_{x} & D 7_{x} & C A_{x} & A D_{x} & 95_{x} & 76_{x} & A 8_{x} \\
A 8_{x} & 2 F_{x} & 49_{x} & D 7_{x} & C A_{x} & A D_{x} & 95_{x} & 76_{x} \\
76_{x} & A 8_{x} & 2 F_{x} & 49_{x} & D 7_{x} & C A_{x} & A D_{x} & 95_{x} \\
95_{x} & 76_{x} & A 8_{x} & 2 F_{x} & 49_{x} & D 7_{x} & C A_{x} & A D_{x}
\end{array}\right) \times\left(\begin{array}{c}
W_{j}[8] \\
W_{j}[9] \\
W_{j}[10] \\
W_{j}[11] \\
U k_{1} \\
W_{j}[13] \\
W_{j}[14] \\
W_{j}[15]
\end{array}\right)=\left(\begin{array}{c}
U k_{2} \\
U k_{3} \\
U k_{4} \\
U k_{5} \\
Z_{j}[12] \\
Z_{j}[13] \\
Z_{j}[14] \\
Z_{j}[15]
\end{array}\right)
$$

Using Inverse Mix Column operation, $Z_{j}[12], Z_{j}[13], Z_{j}[14]$ and $Z_{j}[15]$ can be written as:

$$
\begin{aligned}
& 2 F_{x} \cdot W_{j}[8] \oplus 49_{x} \cdot W_{j}[9] \oplus D 7_{x} \cdot W_{j}[10] \oplus C A_{x} \cdot W_{j}[11] \oplus A D_{x} \cdot U k_{1} \oplus 95_{x} \cdot W_{j}[13] \oplus 76_{x} \cdot W_{j}[14] \oplus A 8_{x} \cdot W_{j}[15]=Z_{j}[12] \\
& A 8_{x} \cdot W_{j}[8] \oplus 2 F_{x} \cdot W_{j}[9] \oplus 49_{x} \cdot W_{j}[10] \oplus D 7_{x} \cdot W_{j}[11] \oplus C A_{x} \cdot U k_{1} \oplus A D_{x} \cdot W_{j}[13] \oplus 95_{x} \cdot W_{j}[14] \oplus 76_{x} \cdot W_{j}[15]=Z_{j}[13] \\
& 76_{x} \cdot W_{j}[8] \oplus A 8_{x} \cdot W_{j}[9] \oplus 2 F_{x} \cdot W_{j}[10] \oplus 49_{x} \cdot W_{j}[11] \oplus D 7_{x} \cdot U k_{1} \oplus C A_{x} \cdot W_{j}[13] \oplus A D_{x} \cdot W_{j}[14] \oplus 95_{x} \cdot W_{j}[15]=Z_{j}[14] \\
& 95_{x} \cdot W_{j}[8] \oplus 76_{x} \cdot W_{j}[9] \oplus A 8_{x} \cdot W_{j}[10] \oplus 2 F_{x} \cdot W_{j}[11] \oplus 49_{x} \cdot U k_{1} \oplus D 7_{x} \cdot W_{j}[13] \oplus C A_{x} \cdot W_{j}[14] \oplus A D_{x} \cdot W_{j}[15]=Z_{j}[15]
\end{aligned}
$$

If we combine the above equations in the following way:

$$
C A_{x} \cdot(E q 22) \oplus A D_{x} \cdot(E q 23) \oplus 49_{x} \cdot(E q 24) \oplus D 7_{x} \cdot(E q 25)
$$

We can eliminate the unknown variable $U k_{1}$ and obtain:

$$
\begin{array}{r}
94_{x} \cdot W_{j}[8] \oplus B 4_{x} \cdot W_{j}[9] \oplus 4 E_{x} \cdot W_{j}[10] \oplus 7 E_{x} \cdot W_{j}[11]=C A_{x} \cdot Z_{j}[12] \oplus A D_{x} \cdot Z_{j}[13] \\
\oplus C 0_{x} \cdot W_{j}[13] \oplus D A_{x} \cdot W_{j}[14] \oplus C 5_{x} \cdot W_{j}[15] \quad \oplus 49_{x} \cdot Z_{j}[14] \oplus D 7_{x} \cdot Z_{j}[15]
\end{array}
$$

\section{Derivation of Eq. 13 defined in Section 5}

$$
\left(\begin{array}{cccccccc}
A D_{x} & 95_{x} & 76_{x} & A 8_{x} & 2 F_{x} & 49_{x} & D 7_{x} & C A_{x} \\
C A_{x} & A D_{x} & 95_{x} & 76_{x} & A 8_{x} & 2 F_{x} & 49_{x} & D 7_{x} \\
D 7_{x} & C A_{x} & A D_{x} & 95_{X} & 76_{x} & A 8_{x} & 2 F_{x} & 49_{x} \\
49_{x} & D 7_{x} & C A_{x} & A D_{x} & 95_{x} & 76_{x} & A 8_{x} & 2 F_{x} \\
2 F_{x} & 49_{x} & D 7_{x} & C A_{x} & A D_{x} & 95_{x} & 76_{x} & A 8_{x} \\
A 8_{x} & 2 F_{x} & 49_{x} & D 7_{x} & C A_{x} & A D_{x} & 95_{x} & 76_{x} \\
76_{x} & A 8_{x} & 2 F_{x} & 49_{x} & D 7_{x} & C A_{x} & A D_{x} & 95_{x} \\
95_{x} & 76_{x} & A 8_{x} & 2 F_{x} & 49_{x} & D 7_{x} & C A_{x} & A D_{x}
\end{array}\right) \times\left(\begin{array}{c}
W_{j}[8] \\
W_{j}[9] \\
W_{j}[10] \\
W_{j}[11] \\
W_{j}[12] \\
W_{j}[13] \\
W_{j}[14] \\
W_{j}[15]
\end{array}\right)=\left(\begin{array}{c}
Z_{j}[8] \\
Z_{j}[9] \\
U k_{1} \\
U k_{2} \\
Z_{j}[12] \\
Z_{j}[13] \\
U k_{3} \\
U k_{4}
\end{array}\right)
$$

Using Inverse Mix Column operation, $Z_{j}[8], Z_{j}[9], Z_{j}[12]$ and $Z_{j}[13]$ can be written as: 
$C A_{x} \cdot W_{j}[8] \oplus A D_{x} \cdot W_{j}[9] \oplus 95_{x} \cdot W_{j}[10] \oplus 76_{x} \cdot W_{j}[11] \oplus A 8_{x} \cdot W_{j}[12] \oplus 2 F_{x} \cdot W_{j}[13] \oplus 49_{x} \cdot W_{j}[14] \oplus D 7_{x} \cdot W_{j}[15]=Z_{j}[9]$ $25_{x} \cdot W_{j}[8] \oplus 49_{x} \cdot W_{j}[9] \oplus D 7_{x} \cdot W_{j}[10] \oplus C A_{x} \cdot W_{j}[11] \oplus A D_{x} \cdot W_{j}[12] \oplus 95_{x} \cdot W_{j}[13] \oplus 76_{x} \cdot W_{j}[14] \oplus A 8_{x} \cdot W_{j}[15]=Z_{j}[12]$ $A 8_{x} \cdot W_{j}[8] \oplus 25_{x} \cdot W_{j}[9] \oplus 49_{x} \cdot W_{j}[10] \oplus D 7_{x} \cdot W_{j}[11] \oplus C A_{x} \cdot W_{j}[12] \oplus A D_{x} \cdot W_{j}[13] \oplus 95_{x} \cdot W_{j}[14] \oplus 76_{x} \cdot W_{j}[15]=Z_{j}[13]$ If we xor together the above four equations, then we get

$$
\begin{aligned}
Z_{j}[8] \oplus Z_{j}[9] \oplus Z_{j}[12] \oplus Z_{j}[13]= & E A_{x} \cdot W_{j}[8] \oplus 54_{x} \cdot W_{j}[9] \oplus 7 D_{x} \cdot W_{j}[10] \oplus C 3_{x} \cdot W_{j}[11] \oplus E 0_{x} \cdot W_{j}[12] \oplus \\
& 5 E_{x} \cdot W_{j}[13] \oplus 7 D_{x} \cdot W_{j}[14] \oplus C 3_{x} \cdot W_{j}[15]
\end{aligned}
$$

